\title{
Frontiers in Multidimensional Self-Trapping of Nonlinear Fields and Matter
}

\author{
Yaroslav V. Kartashov ${ }^{1,2}$, Gregory E. Astrakharchik ${ }^{3}$, Boris A. Malomed ${ }^{4,5,6,7}$, and \\ Lluis Torner ${ }^{1,8}$ \\ ${ }^{1}$ ICFO-Institut de Ciencies Fotoniques, The Barcelona Institute of Science and Technology, 08860 \\ Castelldefels (Barcelona), Spain \\ ${ }^{2}$ Institute of Spectroscopy, Russian Academy of Sciences, Troitsk, Moscow, 108840, Russia \\ ${ }^{3}$ Departamento de Física, Universitat Politècnica de Catalunya, 08034 Barcelona, Spain \\ ${ }^{4}$ Department of Physical Electronics, School of Electrical Engineering, Faculty of Engineering, Tel \\ Aviv University, P.O.B. 39040, Ramat Aviv, Tel Aviv, Israel \\ ${ }^{5}$ Center for Light-Matter Interaction, Tel Aviv University, POB 39040, Ramat Aviv, Tel Aviv, Israel \\ ${ }^{6}$ ITMO University, St. Petersburg 197101, Russia \\ ${ }^{7}$ School of Physics and Optoelectronic Engineering, Foshan University, Foshan 528000, China \\ ${ }^{8}$ Universitat Politècnica de Catalunya, 08034 Barcelona, Spain
}

\begin{abstract}
D and 3D solitons and related states, such as quantum droplets, can appear in optical systems, atomic Bose-Einstein condensates (BECs), and liquid crystals among others. However, multi-dimensional solitary states supported by the standard cubic nonlinearity, tend to be strongly unstable - a property far less present in 1D systems. Thus, the central challenge is to stabilize multi-dimensional states and numerous approaches have been proposed over the years. Most strategies involve noncubic nonlinearities or using various potentials, including periodic ones. Completely new directions have recently emerged in two-component BECs with spin-orbit coupling, which have been predicted to support stable 2D and metastable 3D solitons. A recent breakthrough is the creation of 3D quantum droplets. These are self-sustained states existing in two-component BECs, stabilized by the quantum fluctuations around the underlying mean-field states. Here we review recent results in this field and outline outstanding current challenges.
\end{abstract}

Key points

- We provide a brief summary of recent theoretical and experimental advances in the study of multidimensional solitons, chiefly in nonlinear optics, ultracold bosonic gases and in liquid-crystal and magnetic media.

- We cover results for fundamental nonlinear modes and for topologically nontrivial states, such as vortex solitons, hopfions, and skyrmions.

- The experimental realization of multidimensional solitons has proven to be more challenging than for 1D solitons due to the propensity to instabilities of 2D- and 3D states, both fundamental and topologically structured. We address different stabilization mechanisms that have been put forward to potentially observe multidimensional solitons, such as competing nonlinearities, linear and nonlinear potentials, spin-orbit coupling, quantum corrections, and dissipative effects.

- Special attention is paid to recent theoretical and experimental results that produced stable 3D solitons in the form of 'quantum droplets' in ultracold bosonic gases, whose stability is secured by a macroscopic effect of quantum fluctuations around mean-field states.

40-word summary

Multidimensional self-trapped states exist in many models of physical systems.

However, they are highly unstable in media with a cubic nonlinearity. We review 
different mechanisms that may stabilize them, including non-Kerr nonlinearities, spin-orbit coupling, and quantum fluctuations, among others.

\section{[H1] Introduction}

Soliton-like bound states - self-sustained localized packets of light or matterwaves in nonlinear media-are known in many areas of physics, including nonlinear photonics (optics, plasmonics, and exciton-polariton condensates), BECs and other types of quantum gases, hydrodynamics of classical fluids and superfluids, plasmas, nonlinear dynamical lattices, superconductors, semiconductors, magnetic materials, electronics, hadron matter (considered in terms of field theory), gravitation and cosmology. [1-3]. But in the vast majority of cases, experimental observations of solitons have only been reported in effectively 1D settings.

Higher-dimensional (2D and 3D) geometries open the way to the observation of a variety of new phenomena such as topological states in the form of vortex rings and vortex tori, that are 2D and 3D solitons, respectively, with embedded integer vorticity, $S$. The wave fronts of such states feature one or several nested phase singularities (in particular, vortex lines in the 3D case), whose topological charge, or winding number, is determined by the total phase, $\varphi=2 \pi S$, acquired along a closed contour surrounding the singularity $[4,5]$.

More sophisticated 3D topological self-sustained states may exist (for example, in the form of hopfions and Skyrmions) that carry two independent topological charges [6]. Such states frequently exhibit closed vortex rings inside the wave field. In particular, hopfions are shaped as twisted toroidal vortex modes, with the phase of the wave function varying both along the vortex ring (which determines the overall vorticity $S$ of the hopfion) and also in the transverse plane, around the ring line (which determines the intrinsic twist, $m$, of the hopfion). Skyrmions have fundamentally important physical realizations as baryons in the classical field theory that arises as a low-energy limit of quantum chromodynamics [7] (the original Skyrme model was introduced in this context). Skyrmions were also predicted in two- [8] and multi[9] component BECs and3D topological modes in the form of Dirac monopoles identified as terminus points of vortex lines have been recently observed in spinor BECs [10]. In addition to the more complex structures, multidimensional solitons also often exhibit richer interactions than their 1D counterparts [11].

However, the creation of soliton states in 2D and, especially, in 3D settings is a long-standing challenge because even the simplest fundamental solutions of the corresponding nonlinear wave equations are often prone to strong instabilities, such as the onset of the spatiotemporal collapse of fundamental solitons [12-14] and spontaneous azimuthal splitting of vortex rings and tori $[15,16]$. Theoretical mechanisms allowing the stabilization of higher-dimensional self-trapped states are known, as described below, but they are rare and many of them have proven to be challenging to realize in practice. In particular, in pure optical settings the identification of materials featuring the proper interplay between dispersion, diffraction, and nonlinearity required for the creation of stable, long-living 3D solitons, or 'light bullets' [17], is still an essentially open problem experimentally.

Earlier approaches to the realization of stabilized multidimensional solitons were reviewed in Ref. [4]. Some of the more recent results were briefly summarized in Ref. [18] and [19]. Some early schemes for the stabilization of 2D and 3D solitons, including solitary vortices, relied on the use of non-Kerr nonlinearities, discreteness, and dissipative media. But several important advances have been recently achieved. In particular, the first examples of stable multidimensional states in ultracold bosonic gases, in the form of quantum droplets, the stability of which is grounded in the LeeHung-Yang corrections to the mean-field settings [20], have been predicted and experimentally created very recently [21-30]. Further advances may be foreseen in the nearest future, as several new concepts and techniques are currently under investigation. 


\section{[H1] Multidimensional states in photonics}

\section{[H2] Uniform media}

Spatiotemporal solitons in dispersive optical media may occur when two fundamental linear effects - group-velocity dispersion (GVD), leading to broadening of the electromagnetic wave packet in time, and diffraction, acting in the transverse directions - are balanced by a focusing nonlinearity. The most common source of such nonlinearity is provided by the Kerr effect (known as the $\chi^{(3)}$ nonlinearity) in dielectric media [1-3]. In general, in the case of bright solitary waves, a necessary condition for the balance between the two effects is anomalous GVD: an increasing group velocity with decreasing wavelength, $\lambda$. In the paraxial approximation, the evolution of the wave packet in uniform Kerr-type nonlinear media is governed by the nonlinear Schrödinger equation (NLSE), also known as the Gross-Pitaevskii equation (GPE) in the context of BECs. In normalized form, the NLSE is written as

$$
i \frac{\partial \psi}{\partial z}=-\frac{1}{2}\left(\frac{\partial^{2} \psi}{\partial \tau^{2}}+\frac{\partial^{2} \psi}{\partial x^{2}}+\frac{\partial^{2} \psi}{\partial y^{2}}\right)-\mathcal{N}(\psi) \psi,
$$

where $\psi$ is the complex field amplitude, $z$ is the propagation distance, $\tau=t-z / V_{g r}$ is the reduced time, $V_{g r}$ is the group velocity of the carrier wave, $(x, y)$ are the transverse coordinates, and $\mathcal{N}(\psi)=|\psi|^{2}$ in pure Kerr nonlinear media. The same model without the GVD term, $\partial^{2} \psi / \partial \tau^{2}$, governs the evolution of $2 \mathrm{D}$ beams in the spatial domain.

The NLSE allows the existence of families of bell-shaped (fundamental) spatiotemporal solitons of the form $\psi=u(\rho) \exp (i k z)$, where $k>0$ is the propagation constant, $\rho=\left(x^{2}+y^{2}+\tau^{2}\right)^{1 / 2}$ is the spatiotemporal radial coordinate, and $u$ is a real function vanishing at $\rho \rightarrow \infty$ as $\rho^{-1} \exp \left[-(2 k)^{1 / 2} \rho\right]$. The $2 \mathrm{D}$ version of such states, with $\rho$ replaced by $r=\left(x^{2}+y^{2}\right)^{1 / 2}$ is known as Townes' soliton (TS) [31], with asymptotic decay $\sim r^{-1 / 2} \exp \left[-(2 k)^{1 / 2} r\right]$ at $r \rightarrow \infty$. Historically, TSs were the first species of solitary waves predicted in nonlinear optics, but they cannot be realized experimentally as they are strongly unstable.

The experimental realization of multidimensional solitons faces two main challenges, which have proven to be difficult to overcome despite significant efforts applied during the last three decades. Firstly, the mathematical soliton solutions must be dynamically stable for a given nonlinearity law, $\mathcal{N}(\psi)$. Secondly, in the case of spatiotemporal states, a suitable material setting that simultaneously exhibits sufficiently strong GVD, ultrafast nonlinear response, and low losses is required, so that diffraction and dispersion effects are comparable and this without using too short pulses (ideally, no shorter than a few picoseconds). The latter condition is essential because various higher-order linear and nonlinear effects, which are not included in Eqn. (1), dramatically increase as pulses become shorter, preventing the balance required for the stationary propagation of solitons.

In focusing media, the stability of fundamental solitons is often determined by the Vakhitov-Kolokolov (VK) stability criterion [32]. It predicts the instability of the solutions with $d E / d k<0$, and provides necessary, but sometimes not sufficient, stability condition $d E / d k>0$, where $E=\iiint_{-\infty}^{\infty}|\psi|^{2} d x d y d \tau$ is the total energy of the spatiotemporal state. In uniform Kerr media the scaling relation $\sim k^{1-D / 2}$, where $D=2,3$ is the space dimension, between the energy and the propagation constant holds [4]. This scaling implies the VK instability of 3D solitons. In the 2D case, which corresponds to the above-mentioned TSs, $E$ does not depend on $k$. In this case, the instability still persists, becoming nonlinear and growing sub-exponentially, in contrast to the exponential instability growth that occurs in the 3D setting. Multidimensional solitons can carry vorticity [33], which makes them additionally vulnerable to the instability that causes their spontaneous azimuthal self-splitting into several fragments. .

One possibility to create stable multidimensional solitons, including light bullets, is provided by models with quadratic $\left(\chi^{(2)}\right)$ nonlinearities, which do support ful- 
ly stable solutions in 2D and 3D geometries [34-38]. Stable spatial 2D fundamental solitons were experimentally observed in Ref. [39].Vortex-carrying solitons are unstable in such media [16], as was observed in experiments showing the splitting of the vortex-ring into several fragments [40]. The existence of stable 3D fundamental spatiotemporal solitons in $\chi^{(2)}$ media was originally pointed out in Ref. [41], and subsequently elaborated in several works (see, in particular, Ref. [42]). 2D spatiotemporal solitons (namely, wave packets with nonlinearity balancing temporal spreading and diffraction in only one transverse spatial direction) were created in a series of experiments using a specific method to generate suitable effective anomalous GVD $[43,44]$. Several potential approaches to generate 3D states, for instance using tandem or metamaterial structures with engineered group velocity, GVD, and nonlinearity have been put forward [45]. They are yet to be experimentally demonstrated.

Another strategy for creating stable multidimensional states relies on the use of materials with saturable or competing nonlinearities, such as $\mathcal{N}(\psi)=|\psi|^{2} /(1+$ $v|\psi|^{2}$ ) or $\mathcal{N}(\psi)=|\psi|^{2}-v|\psi|^{4}$ (with real coefficient $v>0$ representing the quintic, alias $\chi^{(5)}$, defocusing nonlinearity), leading to new forms of the $E(k)$ dependencies. Saturable nonlinearity is typical for photorefractive crystals, where a nonlinear change of the refractive index is induced via the electro-optic effect (for a review about its application to spatial solitons, see Ref. [46]), in vapors of alkali metals [47] in particular, sodium vapors [48] - and in other media. The use of saturable nonlinearities enables the creation of a variety of stable 2D spatial solitons. Among them are solitons supported by quasi-steady-state [49] and steady-state screening [50,51], which were observed in photorefractive crystals at low power levels. In Ref. [52] it was shown theoretically that the saturable nonlinearity leads to nonmonotonic $E(k)$ curves. Their segments that satisfy the VK criterion correspond to stable states.

Standard saturable nonlinearities do not suppress azimuthal instabilities of solitons carrying vorticity [53], but competing nonlinearities have been predicted to support stable vortex solitons in both 2D (Ref. [54,55]) and 3D (Ref. [38,56]) geometries. Stability is achieved close to the regime where the contributions from the $\chi^{(3)}$ and $\chi^{(5)}$ nonlinearities almost balance each other, leading to flat-top solitons. These may be described as exhibiting 'liquid-like' behavior [57]. The formation of 2D fundamental and vortex solitons, attributed in part to effective competing $\chi^{(3)}-\chi^{(5)}$ nonlinearities, was experimentally reported in $\mathrm{CS}_{2}$ (Ref. $[58,59]$ ) and in suspensions of metallic nanoparticles [60]. However, the presence of significant linear and nonlinear absorption in such media must be taken into account in the interpretation of the observations and it severely limits the applicability of such materials in the present context.

The competition between nonlinearities of different orders is one of the mechanisms supporting stable dissipative solitons in models of laser cavities, based on the complex Ginzburg-Landau equation $[61,62]$. This is an extension of Eqn. (1) with $\mathcal{N}(\psi)=i \gamma+(1-i \varepsilon)|\psi|^{2}-(v-i \mu)|\psi|^{4}$, which includes linear loss $\gamma$, nonlinear gain $\varepsilon$, and nonlinear absorption $\mu$. Further, spectral filtering is represented by the coefficient $\beta \geq 0$ included into the $(1+i \beta) \partial^{2} \psi / \partial \tau^{2}$ expression which replaces the GVD term in Eqn. (1). Complex Ginzburg-Landau models give rise to stable 3D fundamental solitons $[63,64]$ and vortex tori [65]. Asymmetrically rotating and precessing 3D vortex solitons [66] and vortex knots [67] have been predicted in media with saturable gain and absorption. Recently, dissipative mode-locked cavity solitons with the pulse duration shorter than the cavity round-trip, which may be considered as isolated 3D objects, were observed in a vertical-cavity surface-emitting laser [68]. Such dissipative settings are promising for the generation of multidimensional solitons because they appear as stable attractors with a broad attraction basin, a property that should facilitate their creation.

The possible existence of stable multidimensional states has also been shown in nonlocal media [69]. The respective nonlinear nonlocal response in Eqn. (1), $\mathcal{N}(\psi)=$ $\int K\left(\mathbf{r}^{\prime}-\mathbf{r}\right)\left|\psi\left(\mathbf{r}^{\prime}\right)\right|^{2} d \mathbf{r}^{\prime}$, at a given point $\mathbf{r}$ depends on the entire spatial intensity profile, where $K(\mathbf{r})$ is the nonlocality kernel that depends on the type of nonlinearity. The nonlocality can smooth out the spatial intensity fluctuations, and thus prevent the development of instabilities driven by the Kerr nonlinearity. Nonlocality is typi- 
cal for example for reorientational [70] and thermal [71] optical nonlinearities that respond to time-averaged intensities. Such a nonlocal response is typically very slow, thus it is insensitive to the pulsed character of the excitation and can be used for spatial self-trapping of pulse-train solitons. Fast Kerr nonlinearity, which acts only around the maximum of each pulse, may be used for the dispersion compensation [72]. A similar mechanism allowed the recent observation of dark (in time)-bright (in space) 3D pulse train solitons in photorefractive crystals [73].

\section{1. [H2] Photonic lattices}

A universal theoretical method for the stabilization of both fundamental and vortex solitons in 2D [74-76] and 3D [77-79] geometries with the Kerr focusing nonlinearity is enabled by the use of spatially periodic potentials, induced by photonic lattices or arrays [80-83]. Such potentials are created by shallow $\left(\delta n \sim 10^{-3}\right)$ modulations of the refractive index of the material in the transverse plane. Light propagation in such media is governed by the modified version of Eqn. (1):

$$
i \frac{\partial \psi}{\partial z}=-\frac{1}{2}\left(\frac{\partial^{2} \psi}{\partial \tau^{2}}+\frac{\partial^{2} \psi}{\partial x^{2}}+\frac{\partial^{2} \psi}{\partial y^{2}}\right)-\mathcal{N}(\psi) \psi+U(x, y) \psi,
$$

where the effective potential $U \sim-\delta n(x, y)$ represents the shape of the periodically modulated refractive-index landscape. Different approaches to the creation of such potentials have been suggested. Among them, two techniques have been well elaborated (for other methods see Refs. [82,83]). The first is the induction of virtual optical lattices in the photorefractive media [84], which allowed the creation of 2D fundamental lattice solitons [81], vortex solitons in square [85,86] and hexagonal [87] lattices, as well as soliton trains [88] and dislocations [89]. The second method is based on the direct laser writing of arrays by femtosecond pulses [90]. Optical induction allows erasing and rewriting lattices in the same sample, whereas laser writing creates permanent periodic waveguiding arrays, with practically any desirable shape [91].

Even though the effective potential $U(x, y)$ in Eqn. (2) is $2 \mathrm{D}$, it was theoretically shown to be sufficient for the stabilization of 3D solitons. This was first suggested in combined discrete-continuous systems [92,93], and then in continuous 3D media [77]. The periodic modulation of the refractive index not only stabilizes the 3D solitons (see Ref. [94] for a description of the physics of soliton formation in periodic media), but also allows controlling the relative strength of diffraction and dispersion, which is an essential ingredient in the creation of 3D states. The first observation of semidiscrete 3D light bullets, albeit in a transient state, was an important milestone. It was performed in a hexagonal fiber-like array with silica cores (Fig. 1(a)) illuminated by focused $150 \mathrm{fs}$ pulses at wavelength $\lambda=1.55 \mu \mathrm{m}$ with peak powers up to $1 \mathrm{MW}$ [95]. The temporal compression of such pulses results in the transient excitation of light bullets with a temporal width of $\approx 25 \mathrm{fs}$. With such a small pulse duration, the Raman effect and self-steeping gradually drive the light bullet below its existence threshold, making the spatiotemporal state a transient object [96]. Vortex light bullets were observed in a similar setting, also in a transient form [97]. In fiber arrays, vortex bullets are composed of several spots (three in Ref. [97]), with vorticity imprinted onto them (in contrast to the conventional ring spatial shape of bullets in uniform media), thus building a vortex structure that can coherently propagate in the array, as illustrated in Fig. 1(b).

In addition to the management of diffraction, the approach to the generation of stable 3D solitons based on the use of linear potentials offers control over the energy intervals where 3D solitons may be stable. Stable bullets can exist not only in periodic, but also in radially symmetric (Figs. 2(a) and (b)) and other types of potentials [98]. They exist in complex $\mathcal{P} \mathcal{T}$-symmetric structures [99] too, with spatially separated and globally balanced gain and loss. There vortex bullets develop complex shapes (Figs. 2(c) and (d)) that depend on the sign of the topological charge [100]. In such systems, the otherwise detrimental effect introduced by linear losses, may be used to shape the beam, and control diffraction. 
If the GVD of the material is normal, rather than anomalous, the medium supports a different type of waves, namely a nonlinear generalization of the X-wave solution of the hyperbolic Schrödinger equation [101,102]. Such waves decay in the spatial domain slower than exponentially and, to be stationary, they should carry infinite energy. Nevertheless, truncated versions were observed in waveguide arrays in 2D Ref. [103] and 3D Ref. [104] configurations. Finally, 3D linear wave packets involving combinations of shapes based on the Bessel and Airy functions can also exhibit localized features, even though their invariance upon propagation is a geometric linear phenomenon rather than a result of self-trapping $[105,106]$.

\section{[H2] Multimode waveguides}

The stabilization mechanism of multidimensional states that relies on the inhomogeneity of the refractive-index landscape has also been actively investigated in multimode optical fibers. Standard optical fibers used in telecommunications and other applications are usually designed for single-mode transmission of infrared light, thus they may be considered as effectively 1D media in the context of selftrapping [107]. Graded-index multimode fibers feature a large transverse area, where nonlinearity can couple the multiple modes, resulting in complex spatiotemporal field dynamics [108-114]. In particular, the nonlinearity-induced locking of duration and location of relatively long pulses in different modes, resulting in the formation of spatio-temporal localized states, was experimentally observed in graded-index multimode optical fibers [110]. A significant contribution to the spatial confinement was provided by linear waveguiding properties, induced by the graded-index profile of the fiber.

In this context, we note that the multiple filamentation and the generation of supercontinuum by short pulses in multimode fibers was shown to be enhanced by the selective excitation of multiple spatial modes [111]. Spatiotemporal soliton oscillations in multimode waveguides may generate multimode dispersive waves in an ultrabroadband spectral range [112]. In the normal-GVD regime, multimode fibers were used to demonstrate nonlinearity-induced cleaning of spatial modal profiles $[113,114]$. These may be seen as the condensation of multimode waves, occurring despite the presence of disorder, and subsequent mode decay due to spatiotemporal instabilities. Despite their complexity multimode systems are promising for the creation of fully 3D self-sustained states propagating over considerable distances in weakly-guiding refractive-index profiles, provided that the conditions for the stable nonlinear locking of pulses propagating in different spatial modes can be achieved.

\section{[H2] Exciton-polaritons}

Self-trapping phenomena are a subject of intense investigation in polariton condensates created in semiconductor microcavities. Polaritons are quasi-particles resulting from the coupling of cavity photons and excitons supported by the quantum wells embedded into the cavity. Their low effective mass, which is about five orders of magnitude smaller than the electron's mass in the strong-coupling regime, allows the formation of BECs at temperatures $\sim 5 \mathrm{~K} \mathrm{[115],} \mathrm{which} \mathrm{is} \mathrm{roughly} \mathrm{eight} \mathrm{orders} \mathrm{of}$ magnitude higher than the critical BEC temperature in atomic gases. Such condensates are dissipative states, therefore their persistence is supported by a resonant or non-resonant pump. A noteworthy feature of this system is the strong nonlinear repulsion of polaritons through the dipole-dipole interaction of the excitonic component, which was used for the experimental observation of dark quasi-solitons and vortices [116,117], as well as bright 1D solitons [118,119].

The available technology for microcavity structuring has enabled the creation of various lattice potentials [120,121], and thus of 1D [122] and 2D [123] gap polariton solitons (see also Refs. [124,125] for a detailed theoretical analysis). Polaritons in a planar resonator feature momentum-dependent linear coupling between the states with positive and negative spins, which is formally analogous to the spin-orbit coupling (SOC) in atomic physics [120]. Combined with the sensitivity to magnetic 
fields, SOC enables the realization of nonlinear polariton topological insulators and lasers $[126,127]$. Being essentially a $2 \mathrm{D}$ system, as the confinement in the remaining dimension is imposed by the microcavity, polaritons offer a unique platform for the experimental exploration of the physics of 2D spatial solitons in non-equilibrium condensates, which can be controlled by adjusting the pump laser and by using complex potential landscapes that may be built in the microcavity. In particular, skyrmion lattices in a plasmonic field have been recently realized in experiment [128].

\section{[H2] Plasma filaments}

Multidimensional transient self-trapped states may appear as a result of the filamentation of high-power laser radiation in solids or gases. Filamentation occurs due to the strong spatiotemporal contraction of the wave packet for laser powers exceeding the self-focusing threshold. The contraction leads to the formation of dynamically evolving 3D wave packets that exhibit a high degree of spatiotemporal localization [129]. The propagation of femtosecond quasi-bullets (namely transient modes that are losing energy through multiphoton ionization) over several centimeters in fused silica was reported in Ref. [130]. The formation of bullet chains due to refocusing was demonstrated in Ref. [131], and the creation of bullet filaments by a single fs pulse was reported in Ref. [132]. Such 3D filaments are usually composed of a localized high-intensity core and a ring-shaped spatiotemporal peripheral structure refueling the core [133]. In Ref. [134] such ring-shaped refueling beam was used to considerably extend the propagation distance of the central filament in air, albeit in the normal-GVD regime. Such an approach, when realized in a suitable material with sufficiently highanomalous GVD, may result in the formation of long-lived 3D filaments. New prospects for the robust long-range propagation of multi-terawatt femtosecond laser pulses in the single-filament regime may become available for long wavelengths (in the mid-infrared, in air) [135].

\section{[H1] Multidimensional states supported by nonlinear potentials}

A radically different theoretical approach to the creation of stable multidimensional solitons, which has been studied in a number of settings [136-142], relies on the use of nonlinear potentials (or pseudopotentials [143]) induced by a defocusing nonlinearity with strength $g(\mathbf{r})$ growing fast enough from the center to periphery. The evolution of excitations in such models is governed by the modified multidimensional NLSE/GPE equation,

$$
i \frac{\partial \psi}{\partial t}=-\frac{1}{2} \nabla^{2} \psi+g(\mathbf{r})|\psi|^{2} \psi .
$$

This model predicts the existence of diverse $D$-dimensional self-trapped modes that although decaying at $r \rightarrow \infty$, are nonlinearizable solutions of Eqn. (3), which means that is the asymptotic form of the stationary solutions, $\psi=e^{-i \mu t} u(r)$, with real chemical potential $\mu>0$, at $r \rightarrow \infty$ is determined by the nonlinear term. In particular, for $g(r)=g_{0} r^{\alpha}$, with $\alpha>D$, the asymptotic form of the solution is $u_{r \rightarrow \infty} \approx$ $\left(\mu / 2 g_{0}\right)^{1 / 2} r^{-\alpha / 2}$ at $r \rightarrow \infty$, for $\mu>0$. Such states can be interpreted as solitons with convergent energy (integral norm), provided that $\alpha>D$, which is a fundamental condition for self-trapping for this class of models. In principle, such 2D and 3D models may be implemented in BECs by means of Feshbach resonances controlled by magnetic [144,145] or optical [146,147] fields with appropriate spatial profiles $[148,149]$. The 2D version of the model may also be realized in optics, provided that the local nonlinearity of the material can be shaped, for instance by adjusting the concentration of nonlinearity-inducing dopants [150], or in hollow photonic-crystal fibers infiltrated with index-matching liquids having different nonlinearities.

Systems with spatially inhomogeneous repulsive nonlinearities of this type have been theoretically shown to support a variety of stable $2 \mathrm{D}$ and, also, 3D soli- 
tons. These can be stable vortex solitons with high values of the topological charge $S$ $[137,140]$, which are usually highly unstable in other systems. In contrast, 3D vortex solitons predicted in these systems were found to be robust. In particular, the sudden application of a torque does not destroy them, but rather casts them into a state of stable precession, thus providing a remarkable example of a vortex-soliton gyroscope [140]. The model in Eqn. (3) also gives rise [141] to stable self-trapped toroidal vortex rings or hopfions [6], which carry the usual topological charge (winding number), $S$, and an intrinsic twist of the torus, characterized by an independent topological charge, $m$. Illustrative examples are shown in Figs. 3(a) and 3(b). States such as hopfions were considered impossible in previously studied scalar (single-field) settings. Hybrid 3D vortex solitons, composed of two vertically separated vortex states with equal $S_{1}=S_{2}=1$ or opposite $S_{1}=-S_{2}=1$ topological charges, may exist in anisotropic 'peanut-shaped' nonlinearity landscapes [142], representing, in the case of $S_{1}=-S_{2}=1$, the first example of $3 \mathrm{D}$ composite vortex-antivortex modes. These are shown in Figs. 3(c) and 3(d). The realization of all these fascinating theoretical predictions depends on the practical feasibility of the models, which is still awaiting experimental confirmation.

\section{[H1] Multidimensional states in atomic BECs}

\section{[H2] Stabilization by optical lattices}

Similar to the situation in nonlinear optics, a universal method for the stabilization of matter-wave multidimensional solitons, including vortical ones, is provided by the use of periodic potentials. These can be induced as optical lattices [151]. However, a fundamentally new feature is the possibility to create 3D potentials. These have been used in many experiments, such as the observation of the Mott-insulator phase [152,153], but, thus far, not for the creation of solitons, despite several important theoretical predictions.

In particular, stable 2D gap solitons, including states with embedded vorticity, were predicted to exists in BECs with repulsive nonlinearity and a square-lattice potential over a decade ago [154-156]. Such solitons feature unique physical properties. For example 2D gap solitons are mobile, with a negative effective mass (which is a generic property of solitons of the gap type $[94,151])$. As a result, an additional trapping harmonic-oscillator potential superimposed on the periodic lattice expels the solitons, while the anti-trapping potential, with the inverted sign, supports stable motion of the gap soliton along an elliptic trajectory [157]. Bright 2D solitons have also been predicted to exist in a stable form in radial lattices, including radial potentials shaped like Bessel functions [158], or those represented by periodic functions of the radial variable, such as $\cos (k r+\delta)$ with wavenumber $k$ and phase shift $\delta$ [159]. Solitons created in such potentials may be strongly localized objects, capable to perform motion along circular trajectories in annular potentials [158]. These and several other related theoretical predictions for multidimensional solitons in BECs are yet to be experimentally demonstrated.

\section{[H2] Stabilization by SOC}

Progress in predicting settings that may support stable 2D and 3D matter-wave solitons has been boosted by the observation of SOC in binary BECs $[160,161]$. A noteworthy result is that SOC has been theoretically shown to create the ground state in the 2D GPE with attractive nonlinearity, in the form of solitons of the SV (semivortex) and MM (mixed-mode) types [162-164]. In the absence of SOC, the free-space GPE in 2D has no ground state (it is formally replaced by the collapsing solution). The evolution of binary BECs under the action of SOC is modelled by the coupled GPEs for the components $\psi_{+}(x, y, t)$ of the mean-field wave function [160-164], which take the following form in $2 \overline{\mathrm{D}}$ : 


$$
\begin{aligned}
& i \frac{\partial \psi_{+}}{\partial t}=-\frac{1}{2} \nabla^{2} \psi_{+}-\left(\left|\psi_{+}\right|^{2}+\gamma\left|\psi_{-}\right|^{2}\right) \psi_{+}+\left(\lambda_{\mathrm{R}} \mathcal{D}^{(-)}-i \lambda_{\mathrm{D}} \mathcal{D}^{(+)}\right) \psi_{-} \\
& i \frac{\partial \psi_{-}}{\partial t}=-\frac{1}{2} \nabla^{2} \psi_{-}-\left(\left|\psi_{-}\right|^{2}+\gamma\left|\psi_{+}\right|^{2}\right) \psi_{-}+\left(\lambda_{\mathrm{R}} \mathcal{D}^{(+)}-i \lambda_{\mathrm{D}} \mathcal{D}^{(-)}\right) \psi_{+}
\end{aligned}
$$

where the SOC operators are $\mathcal{D}^{( \pm)}=\partial / \partial x \pm i \partial / \partial y$, with real coefficients $\lambda_{\mathrm{R}, \mathrm{D}}$ for the SOC terms of the Rashba and Dresselhaus types, respectively, and $\gamma$ is the relative strength of the cross-attraction between the two components.

In particular, solitons of the SV type with chemical potential $\mu$ are represented, in terms of the polar coordinates, by an ansatz which is compatible with Eqn. (4): $\psi_{+}=e^{-i \mu t} \phi_{+}(r), \psi_{-}=e^{-i \mu t+i \theta} r \phi_{-}(r)$, where functions $\phi_{ \pm}(r)$ are finite at $r=0$, decaying as $\sim \exp \left[-(-2 \mu)^{1 / 2} r\right]$ at $r \rightarrow \infty$. The SV component $\psi_{-}$is the vortical one, whereas $\psi_{+}$carries zero vorticity (hence its semi-vortex nature).

An essential effect introduced by SOC is the breaking of the scaling invariance of the 2D GPE, which is the source of the above-mentioned degeneracy of the TS (which means that all TS solutions have the single value of the norm). As a result, the coupled GPEs in Eqn. (4) give rise to a family of solitons with values of the norm that completely fill the interval from 0 up to norm $N_{\mathrm{T}}$ of the degenerate family of TSs, as shown in Fig. 4(a). Because $N_{\mathrm{T}}$ is the threshold necessary for the onset of the critical collapse, solutions whose norm falls below $N_{\mathrm{T}}$ cannot initiate collapse, which secures their stability. Thus, SVs become the ground state that is missing in the usual selfattractive GPE in 2D, as mentioned above. Furthermore, the entire SV branch satisfies the VK stability criterion, $d \mu / d N<0$. Both the SVs and MMs exist at all values of parameters, but the SVs are stable at $\gamma \leq 1$ and unstable at $\gamma \geq 1$, and the MMs are predicted to be stable exactly in the opposite case. The physical origin of this phenomenon arises from the fact that the SV and MM species realize the energy minimum at $\gamma \leq 1$ and $\gamma \geq 1$, respectively [162]. Increasing the Dresselhaus coefficient in Eqn. (4), $\lambda_{\mathrm{D}}$, while keeping $\lambda_{\mathrm{R}}=1$, eventually leads to delocalization (disappearance of solitons) at a critical point [164].

Soliton mobility in the framework of Eqn. (4) is a nontrivial issue, as SOC breaks the Galilean invariance of the usual GPE. Numerical analysis has shown that MMs exhibit mobility in one direction ( $y$ in Eqn. (4)), up to a critical value of the velocity, beyond which the soliton's amplitude vanishes. For the SVs, the critical velocity was found to be extremely small [162].

Regarding 3D states, SOC cannot suppress the supercritical collapse. Nevertheless, the 3D generalization of Eqn. (4) has been shown numerically to give rise to 3D metastable soliton states, which realize local energy minima, being therefore stable against small perturbations [165]. As well as in the 2D geometries, 3D systems give rise to metastable solitons of the SV and MM types at $\gamma \leq 1$ and $\gamma \geq 1$, respectively, as illustrated in Figs. 4(b) and 4(c). All results described in this section are awaiting experimental exploration.

\section{[H2] Giant vortex solitons in binary BECs}

Theoretical efforts have been devoted to elucidate a physically realizable BEC model that may support stable vortex solitons with high values of vorticity $S$. Note that, in SOC systems, all such states have been shown to be unstable [162]. A relevant 2D model, which produces stable vortex solitons with high values of $S$ was put forward in Ref. [166], in the form of coupled GPEs for wave functions representing two different hyperfine atomic states in an ultracold bosonic gas, resonantly coupled by a magnetic component, $H$, of a microwave electromagnetic field. In turn, the microwave field is generated by the Poisson equation with the respective source density, $\psi_{+} \psi_{-}^{*}$. Eliminating the magnetic field by means of the Green function of the 2D Poisson equation, the corresponding governing equation can be cast in the form featuring an effectively nonlocal interaction (possibly combined with the local nonlinearity):

$$
i \frac{\partial \psi_{ \pm}}{\partial t}=-\frac{1}{2} \nabla^{2} \psi_{ \pm}-\beta\left|\psi_{ \pm}\right|^{2} \psi_{ \pm}+\Gamma \psi_{\mp} \int \psi_{ \pm}\left(\mathbf{r}^{\prime}\right) \psi_{\mp}^{*}\left(\mathbf{r}^{\prime}\right) \ln \left|\mathbf{r}-\mathbf{r}^{\prime}\right| d \mathbf{r}^{\prime}
$$


where $\Gamma$ is the strength of the feedback of the wave function onto the magnetic field, and $\beta$ is the strength of the contact self-interaction of the BEC. Solving Eqn. (5) numerically produces vortex solitons which are predicted to be stable, at least, up to $S=5$ (hence the term 'giant vortices') [166]. Illustrative examples are shown in Fig. 5. Similar to the above-mentioned exciton-polariton system, these are hybrid solitons, as they include both the matter-wave and field components. Their experimental observation is still an open question.

\section{[H2] Stable quantum droplets in binary BECs}

A recent breakthrough opens up a whole new direction in multidimensional self-trapping. This is the creation of a new class of quantum liquids [24-29], which are fully coherent and extremely diluted (their density is of the order of $\sim 10^{14}$ atoms $/ \mathrm{cm}^{3}$, which is eight orders of magnitude smaller than that of liquid helium [24]). Already in 2002, it was pointed out [167] that bosonic droplets (or 'boselets') could in principle be created if the mean-field (MF) energy of self-attraction is compensated by three-body repulsive terms, resulting in a minimum of the energy as a function of density, although such mechanism was not used in practice. Then, in 2015 it was predicted that the repulsive Lee-Hung-Yang (LHY) corrections [20], induced by quantum fluctuations around the MF state, can be exploited for the stabilization of the two-component condensates [21,22] (see also Ref. [168]). In 3D geometries, the interactions should be tuned in such a way that the self-repulsion in each component is slightly overbalanced by the attraction between the components [21]. Note that the strength of interactions can be tuned by exploiting a Feshbach resonance. Such systems would be unstable in models based solely on the MF approximation, but the LHY corrections can make them stable.

Unlike classical liquids, in which the equilibrium density is uniquely determined by the interaction potential (typically of the Van der Waals type), in quantum droplets it is controlled by the balance of the MF and LHY terms, and thus can be tuned [169]. The diluteness of the quantum droplets justifies the applicability of perturbation theory, in contrast to usual quantum liquids where such an approach is not valid. The separation of scales of the underlying soft and hard modes allows the derivation of the LHY terms in local form, depending solely on the density. For a mixed binary fluid with equal wave functions $\psi$ of the two components, this implies addition of a self-repulsive quartic term with coefficient $g_{\mathrm{LHY}}>0$ to the 3D GPE with the effective MF cubic self-attraction, with respective coefficient $g>0$ Ref. [21], as:

$$
i \frac{\partial \psi}{\partial t}=-\frac{1}{2} \nabla^{2} \psi-g|\psi|^{2} \psi+g_{\mathrm{LHY}}|\psi|^{3} \psi .
$$

Exact Monte-Carlo calculations $[22,172,173,174]$ and approximate variational estimates [175] have been found to agree with the predictions of Eqn. (6) in the limit of weak interactions. For stronger interactions the corrections due to the finite effective range can be significant $[174,175]$, thus an empiric energy functional taking into account such effects was proposed [174]. Modifications of the system for Rabi-coupled [170] and SOC-coupled [30] mixtures, as well as Bose-Fermi systems [171], were recently elaborated. The reduction of the 3D model in Eqn. (6) to lower dimensions, under the action of tight confinement, has been reported [22]. In the case of equal wave functions of the two components as described by Eqn. (6), the 2D model amounts to the single GPE with a nonlinear term $\sim|\psi|^{2} \psi \ln |\psi|^{2}$, which implies selfattraction for small densities $|\psi|^{2}$, and repulsion for large $|\psi|^{2}$. Such nonlinearity has been theoretically shown to give rise to stable fundamental states [22,30], as well as to states with embedded vorticity with topological charge up to $S=5$ Ref. [176]. The $1 \mathrm{D}$ geometry is special in that the beyond-MF correction turns out to be effectively attractive, $\sim-|\psi| \psi$, thus the quantum liquid is formed when the MF term is slightly repulsive $[22,177]$. 
Ultradilute quantum droplets have been realized experimentally recently [2426]. Single-component dipolar condensates [23,178-184] exhibit a competition between short-range and anisotropic long-range interactions. The interactions are tuned in such a way that the MF energy is slightly negative, whereas the repulsive LHY corrections stabilize the system [181,182] against collapse [183]. Unlike fundamental dipolar quantum droplets, their counterparts with embedded vorticity were found to be unstable [184].

Stable single-component dipolar quantum droplets were created recently in condensates of ${ }^{164}$ Dy Ref. [24,25] and ${ }^{166}$ Er Ref. [26]. It was experimentally verified that the stabilization mechanism indeed originates from quantum fluctuations [25]. Due to the nature of the dipole-dipole interactions, the dipolar quantum droplets feature strong anisotropy, as shown by the 3D simulations illustrated in Fig. 6(a). The creation of 3D states in gases with contact interactions was recently demonstrated experimentally [27-29], using binary mixtures of two hyperfine atomic states of ${ }^{39} \mathrm{~K}$. In these experiments, the necessary balance between the repulsive and attractive intra- and inter-component interactions was achieved by means of the Feshbach resonance. The experiments were performed both in the presence of a strong confining potential, applied in one direction, which leads to the formation of quasi-2D states $[27,28]$, and in the absence of the confinement, which allowed the observation of isotropic droplets [29]. The boundary between the quantum-droplet and usual soliton regimes was experimentally mapped in Ref. [28]

Recently stable quantum droplets with embedded vorticity in the full 3D model have been theoretically predicted [185]. The corresponding solution to Eqn. (6) with chemical potential $\mu$ and integer vorticity $S$ has the form of $\psi=\exp (-i \mu t+$ $i S \theta) \phi(\rho, z)$ in cylindrical coordinates $(z, \rho, \theta)$, where the real function $\phi(\rho, z)$ decays exponentially at $\rho \rightarrow \infty$ and $|z| \rightarrow \infty$, and vanishes as $\sim \rho^{|S|}$ at $\rho \rightarrow 0$. The family of vortical states with $S=1$ was found to be partly stable and a narrow stability region was also found to exist for $S=2$. It was also found that vortex solitons are stable when the norm exceeds a minimum value which scales as $\sim S^{6}$, which explains the difficulty of finding stable modes with $S>1$ (in the 2D model, with the abovementioned nonlinearity, $\sim|\psi|^{2} \psi \ln |\psi|^{2}$, the corresponding scaling is $S^{4}$, making it possible to construct stable states up to $S=5$ Ref. [176]).

Estimates indicate that the experimental creation of such states in a mixed condensate ${ }^{87} \mathrm{Rb}-{ }^{41} \mathrm{~K}$ requires densities of the order of $\sim 5 \times 10^{15}$ atoms $/ \mathrm{cm}^{3}$. Reaching such states experimentally is challenging at present, but they should become achievable as the state of the art advances.

\section{[H1] Multidimensional solitons in liquid crystals and liquid ferromagnets}

Recent experiments in liquid crystals and in ferrofluids, made of colloidal suspensions of disk-shaped magnetic nanoparticles [186-188] have allowed the study of static material structures (rather than propagating fields discussed in all previous sections). Topologically structured 3D solitons were created, such doughnut-shaped torons (twisted cylinders closed on themselves in the form of tori similar to the above-mentioned hopfions in the BEC model), which coupled to the surrounding uniform field, as illustrated by Figs. 7(a)-7(c). In spite of the mathematical similarity to the matter-wave hopfions, the nature of these $3 \mathrm{D}$ states is drastically different from those addressed in previous sections as they occur in completely different physical settings, as explained below.

In nematic liquid crystals, the hopfions in the form of torons are realized as finite-energy configurations of the corresponding order parameter (director field), characterized by an intrinsic knotted structure, with different particular knotted configurations identified by values of the corresponding Hopf topological number, which represents different types of states [186]. Each particular type may be characterized by the so-called pre-image: a closed loop in the 3D space, which exhibits the same knottedness as carried by the respective toron in the liquid-crystal medium. In Ref. [186], various species of the torons were created experimentally, using laser tweezers to set the necessary configurations of the order parameter, and an optical 
imaging technique was employed to observe the shapes of the respective 3D states. The actual size of the modes created in the nematic samples is measured on the micron scale. Thus, pre-images of these 3D toron modes were reconstructed from the experimental data and reproduced numerically using the Hamiltonian of the nematic liquid crystals, expressed in terms of the local order parameter. As stated by the Hobart-Derrick theorem $[189,190]$, rigorous energy arguments demonstrate that 3D solitons, built of a single real scalar field, cannot be stable, unless the underlying Hamiltonian density includes higher-order spatial derivatives, in addition to the standard squared-gradient term. The stability of torons/hopfions found in Ref. [186] stems from the fact that they are based on the vectorial order parameter.

Similar experimental and theoretical results were reported in Ref.[188] for hopfions created in liquid-colloidal ferromagnets, in which the same role as the local director in the above-mentioned liquid crystals is played by the local direction of the magnetization (whereas its absolute value is fixed), the respective Hamiltonian being similar to its liquid-crystal counterpart. We also mention the recent experimental creation of a 3D stable nontopological soliton, in the form of a confined region of oscillating nematic director. It propagates in the liquid crystal at a constant velocity, in the presence of dc electric field, perpendicular to the field and to the original orientation of the director [191]. Topological modes, such as Skyrmions in magnetic media [192] and nematic liquid crystals [193], may also move, in principle with much larger speeds than the nontopological solitons.

Despite the rather specific nature of these systems, they are important as a way to experimentally construct distinct species of fully macroscopic and perfectly stable 3D self-trapped topologically organized states (and non-topological 3D solitons as well) under well-controlled conditions. In addition, the use of well-established underlying Hamiltonians makes it possible to accurately identify the observed modes as expected complex topological states. An example of the cross-fertilization between different areas is the potential existence of 3D structures defined by knotted core lines of the mean-field wave-function patterns, recently predicted for BECs loaded into an optical lattice [194].

\section{[H1] Conclusion}

This paper presents a brief overview of the recent progress in the creation of dynamically stable multidimensional soliton-like states, with a focus on optical materials, matter-wave condensates and ultradilute quantum liquids, liquid crystals and ferrofluids. Despite not being exhaustive, the Review aims at bridging different communities in an attempt to foster the exchange of ideas and stimulate the implementation of concepts, originally developed in a specific physical setting, in completely different ones. The motivation is harnessing the unique opportunities offered by multidimensional settings for the creation of localized states with rich internal structures that are not possible in 1D geometries.

The conclusion drawn from more than three decades worth of efforts is that theoretical predictions are far more advanced than experimental demonstrations. Various 2D states (both spatial and spatio-temporal ones) have been observed experimentally, but robust 3D states are more difficult to create. The main challenge to be overcome is the trend of multidimensional states to be strongly unstable. This is in sharp contrast to 1D solitary waves, which exist as robust states in many systems and may be used in practical applications. An example is the recent use of dissipative Kerr temporal solitons for the generation of robust frequency combs that are of paramount importance to high-precision techniques in a variety of scientific and technological areas [195].

A recent breakthrough is the experimental creation of quantum droplets that are stable in 2D and 3D geometries due to the effects of quantum fluctuations. Beyond their intrinsic significance, these observations open up an important direction for exploration of concepts directly linked to multidimensionality. Along these lines, we note that over the past decade, photonic settings and BECs in optical lattices proved to be powerful tools to mimic the behaviour of other physical systems that 
are much harder to access experimentally. The research about the formation of multidimensional self-trapped states should be seen from a similar perspective, hence its fundamental and far-reaching importance.

\section{Author contributions}

All authors contributed to all sections of the paper. Section IV was chiefly written by BAM.

\section{Acknowledgments}

We greatly appreciate many valuable collaborations and discussions with Sadhan K. Adhikari, Guangjiong Dong, Arnaldo Gammal, Randall G. Hulet, Vladimir V. Konotop, Oleg D. Lavrentovich, Yongyao Li, Dumitru Mihalache, Dmitry S. Petrov, Hidetsugu Sakaguchi, Luca Salasnich, Evgeny Ya. Sherman, Yasha Shnir, Dmitry V. Skryabin, and Leticia Tarruell.

L.T. and Y.V.K. acknowledge support from the Severo Ochoa program (SEV-20150522) of the Government of Spain, Fundacio Cellex, Fundació Mir-Puig, Generalitat de Catalunya and CERCA. The work of B.A.M. is supported, in part, by the joint program in physics between NSF and Binational (US-Israel) Science Foundation through project No. 2015616, and by Israel Science Foundation through Grant No. 1286/17. This author appreciates hospitality of ICFO during the preparation of this review. G.E.A. acknowledges financial support from the MICINN (Spain) Grant No FIS2017-84114-C2-1-P.

Publisher's note

Springer Nature remains neutral with regard to jurisdictional claims in published maps and institutional affiliations.

\section{References:}

[1] N. N. Akhmediev and A. Ankiewicz, Solitons: Nonlinear Pulses and Beams, Chapman \& Hall, 1997.

[2] Y. S. Kivshar and G. P. Agrawal, Optical Solitons: From Fibers to Photonic Crystals, Academic Press: San Diego, 2003.

[3] T. Dauxois and M. Peyrard, Physics of Solitons, Cambridge University Press, Cambridge, 2006.

[4] B. A. Malomed, D. Mihalache, F. Wise, L. Torner, Spatiotemporal optical solitons, J. Optics B 7, R53-R72 (2005).

[5] D. Mihalache, Multidimensional localized structures in optical and matter-wave media: A topical review of recent literature, Rom. Rep. Phys. 69, 403 (2017).

[6] E. Radu and M. S. Volkov, Stationary ring solitons in field theory - knots and vortons, Phys. Rep. 468, 101-151 (2008).

[7] M. Bender, P.-H. Heenen, and P.-G. Reinhard, Self-consistent mean-field models for nuclear structure, Rev. Mod. Phys. 75, 121-180 (2003).

[8] J. Ruostekoski, Stable particlelike solitons with multiply quantized vortex lines in BoseEinstein condensates, Phys. Rev. A 70, 041601(R) (2004).

[9] K. Tiurev, T. Ollikainen, P. Kuopanportti, M. Nakahara, D. S. Hall, and M. Möttönen, Three-dimensional skyrmions in spin-2 Bose-Einstein condensates, New J. Phys. 20, 055011 (2018).

[10] M. W. Ray, E. Ruokokoski, S. Kandel, M. Möttönen, and D. S. Hall, Observation of Dirac monopoles in a synthetic magnetic field, Nature 505, 657-660 (2014).

[11] J. H. V. Nguyen, P. Dyke, D. Luo, B. A. Malomed, R. G. Hulet, Collisions of matterwave solitons, Nature Phys. 10, 918-922 (2014). 
[12] L. Berge, Wave collapse in physics: Principles and applications to light and plasma waves, Phys. Rep. 303, 259-372 (1998).

[13] E. A. Kuznetsov and F. Dias, Bifurcations of solitons and their stability, Phys. Rep. 507, 43-105 (2011).

[14] G. Fibich, The Nonlinear Schrödinger Equation: Singular Solutions and Optical Collapse (Springer, Heidelberg, 2015).

[15] W. J. Firth and D. V. Skryabin, Optical solitons carrying orbital angular momentum, Phys. Rev. Lett. 79, 2450-2453 (1997).

[16] L. Torner and D. V. Petrov, Azimuthal instabilities and self-breaking of beams into sets of solitons in bulk second-harmonic generation, Electron. Lett. 33, 608-610 (1997).

[17] Y. Silberberg, Collapse of optical pulses, Opt. Lett. 22, 1282-1284 (1990).

[18] B. A. Malomed, D. Mihalache, F. Wise, and L. Torner, Viewpoint: On multidimensional solitons and their legacy in contemporary Atomic, Molecular and Optical physics, J. Phys. B: At. Mol. Opt. Phys. 49, 170502 (2016).

[19] B. A. Malomed, Multidimensional solitons: Well-established results and novel findings, Eur. Phys. J. Special Topics 225, 2507-2532 (2016).

[20] T. D. Lee, K. Huang, C. N. Yang, Eigenvalues and eigenfunctions of a Bose system of hard spheres and its low-temperature properties, Phys. Rev. 106, 1135-1145 (1957).

[21] D. S. Petrov, Quantum mechanical stabilization of a collapsing Bose-Bose mixture, Phys. Rev. Lett. 115, 155302 (2015).

[22] D. S. Petrov and G. E. Astrakharchik, Ultradilute low-dimensional liquids, Phys. Rev. Lett. 117, 100401 (2016).

[23] D. Baillie, R. M. Wilson, R. N. Bisset, P. B. Blakie, Self-bound dipolar droplet: A localized matter wave in free space, Phys. Rev. A 94, 021602(R) (2016).

[24] M. Schmitt, M. Wenzel, B. Böttcher, I. Ferrier-Barbut, and T. Pfau, Self-bound droplets of a dilute magnetic quantum liquid, Nature 539, 259-262 (2016).

[25] I. Ferrier-Barbut, H. Kadau, M. Schmitt, M. Wenzel, T. Pfau, Observation of quantum droplets in a strongly dipolar Bose gas, Phys. Rev. Lett. 116, 215301 (2016).

[26] L. Chomaz, S. Baier, D. Petter, M. J. Mark, F. Wächtler, L. Santos, F. Ferlaino, Quantum-fluctuation-driven crossover from a dilute Bose-Einstein condensate to a macrodroplet in a dipolar quantum fluid, Phys. Rev. X 6, 041039 (2016).

[27] C. R. Cabrera, L. Tanzi, J. Sanz, B. Naylor, P. Thomas, P. Cheiney, L. Tarruell, Quantum liquid droplets in a mixture of Bose-Einstein condensates, Science 359, 301-304 (2018).

[28] P. Cheiney, C. R. Cabrera, J. Sanz, B. Naylor, L. Tanzi, and L. Tarruell, Bright Soliton to Quantum Droplet Transition in a Mixture of Bose-Einstein Condensates, Phys. Rev. Lett. 120, 135301 (2018).

[29] G. Semeghini, G. Ferioli, L. Masi, C. Mazzinghi, L. Wolswijk, F. Minardi, M. Modugno, G. Modugno, M. Inguscio, and M. Fattori, Self-bound quantum droplets in atomic mixtures, Phys. Rev. Lett. 120, 235301 (2018).

[30] Y. Li, Z. Luo, Y. Liu, Z. Chen, C. Huang, S. Fu, H. Tan, and B. A. Malomed, Twodimensional solitons and quantum droplets supported by competing self-and crossinteractions in spin-orbit-coupled condensates, New J. Phys. 19, 113043 (2017).

[31] R. Y. Chiao, E. Garmire, and C. H. Townes, Self-trapping of optical beams, Phys. Rev. Lett. 13, 479-482 (1964).

[32] M. Vakhitov and A. Kolokolov, Stationary solutions of the wave equation in a medium with nonlinearity saturation, Radiophys. Quantum Electron. 16, 783-789 (1973).

[33] V. I. Kruglov and R. A. Vlasov, Spiral self-trapping propagation of optical beams in media with cubic nonlinearity, Phys. Lett. A 111, 401-404 (1985).

[34] Yu. N. Karamzin and A. P. Sukhorukov, Mutual focusing of high-power light beams in media with quadratic nonlinearities, Sov. Phys. JETP. 41, 414-420 (1976).

[35] G. I. Stegeman, D. J. Hagan, and L. Torner, Cascading phenomena and their applications to all-optical signal processing, mode-locking, pulse compression and solitons, Opt.

Quant. Electr. 28, 1691-1740 (1996).

[36] C. Etrich, F. Lederer, B. A. Malomed, T. Peschel, and U. Peschel, Optical solitons in media with a quadratic nonlinearity, Progress in Optics 41, 483-568 (2000). 
[37] A. V. Buryak, P. Di Trapani, D. V. Skryabin, and S. Trillo, Optical solitons due to quadratic nonlinearities: from basic physics to futuristic applications, Phys. Rep. 370, 63235 (2002).

[38] D. Mihalache, D. Mazilu, L.-C. Crasovan, I. Towers, A. V. Buryak, B. A.

Malomed, L. Torner, J. P. Torres, F. Lederer, Stable spinning optical solitons in three dimensions, Phys. Rev. Lett. 88, 073902 (2002).

[39] W. E. Torruellas, Z. Wang, D. J. Hagan, E. W. van Stryland, G. I. Stegeman, L. Torner, and C. R. Menyuk, Observation of two-dimensional spatial solitary waves in a quadratic medium, Phys. Rev. Lett. 74, 5036-5039 (1995).

[40] D.V. Petrov, L. Torner, J. Martorell, R. Vilaseca, J. P. Torres, C. Cojocaru, Observation of azimuthal modulational instability and formation of patterns of optical solitons in a quadratic nonlinear crystal, Opt. Lett. 23, 1444-1446 (1998).

[41] A. A. Kanashov and A. M. Rubenchik, On diffraction and dispersion effects on threewave interaction, Physica D 4, 122-134 (1981).

[42] B. A. Malomed, P. Drummond, H. He, A. Berntson, D. Anderson, and M. Lisak, Spatio-temporal solitons in optical media with a quadratic nonlinearity, Phys. Rev. E 56, 4725-4735 (1997).

[43] X. Liu, L. J. Qian, F. W. Wise, Generation of optical spatiotemporal solitons, Phys. Rev. Lett. 82, 4631-4634 (1999).

[44] X. Liu, K. Beckwitt, and F. W. Wise, Two-dimensional optical spatiotemporal solitons in quadratic media, Phys. Rev. E 62, 1328-1340 (2000).

[45] L. Torner, S. Carrasco, J. P. Torres, L.-C. Crasovan, and D. Mihalache, Tandem light bullets, Opt. Commun. 199, 277-281 (2001).

[46] Z. Chen, M. Segev, and D. N. Christodoulides, Optical spatial solitons: historical overview and recent advances, Rep. Prog. Phys. 75, 086401 (2012).

[47] V. Tikhonenko, J. Christou, and B. Luther-Davies, Three-dimensional bright spatial soliton collision and fusion in a saturable nonlinear medium, Phys. Rev. Lett. 76, 2698-2701 (1996).

[48] J. Bjorkhol and A. Ashkin, CW self-focusing and self-trapping of light in sodium vapour, Phys. Rev. Lett. 32, 129-132 (1974).

[49] G. C. Duree, J. L. Shultz, G. J. Salamo, M. Segev, A. Yariv, B. Crosignani, P. Di Porto, E. J. Sharp, and R. R. Neurgaonkar, Observation of self-trapping of an optical beam due to the photorefractive effect, Phys. Rev. Lett. 71, 533-536 (1993).

[50] M. D. Iturbe Castillo, P. A. Marquez Aguilar, J. J. Sanchez-Mondragon, S. Stepanov, and V. Vysloukh, Spatial solitons in photorefractive BTO with drift mechanism of nonlinearity, Appl. Phys. Lett. 64, 408-410 (1994).

[51] M. Segev, G. C. Valley, B. Crosignani, P. Di Porto, and A. Yariv, Steady-state spatial screening solitons in photorefractive materials with external applied-field, Phys. Rev. Lett. 73, 3211-3214 (1994).

[52] D. E. Edmundson and R. H. Enns, Robust bistable light bullets, Opt. Lett. 17, 586588 (1992).

[53] J. M. Soto-Crespo, D. R. Heatley, E. M. Wright, and N. N. Akhmediev, Stability of the higher-bound states in a saturable self-focusing medium, Phys. Rev. A 44, 636-644 (1991).

[54] M. Quiroga-Teixeiro and H. Michinel, Stable azimuthal stationary state in quintic nonlinear media, J. Opt. Soc. Am. B 14, 2004-2009 (1997).

[55] M. L. Quiroga-Teixeiro, A. Berntson, and H. Michinel, Internal dynamics of nonlinear beams in their ground states: short- and long-lived excitation, J. Opt. Soc. Am. B 16, 1697-1704 (1999).

[56] A. Desyatnikov, A. Maimistov, and B. Malomed, Three-dimensional spinning solitons in dispersive media with the cubic-quintic nonlinearity, Phys. Rev. E 61, 3107-3113 (2000).

[57] A. Paredes, D. Feijoo, and H. Michinel, Coherent cavitation in the liquid of light, Phys. Rev. Lett. 112, 173901 (2014).

[58] E. L. Falcão-Filho, C. B. de Araújo, G. Boudebs, H. Leblond, and V. Skarka, Robust two-dimensional spatial solitons in liquid carbon disulfide, Phys. Rev. Lett. 110, 013901 (2013). 
[59] S. Reyna, G. Boudebs, B. A. Malomed, and C. B. de Araújo, Robust self-trapping of vortex beams in a saturable optical medium, Phys. Rev. A 93, 013840 (2016).

[60] A. S. Reyna, K. C. Jorge, and C. B. de Araújo, Two-dimensional solitons in a quinticseptimal medium, Phys. Rev. A 90, 063835 (2014).

[61] N. Akhmediev and A. Ankiewicz, Dissipative Solitons, Lecture Notes in Physics, vol. 661 (Springer, 2005).

[62] P. Grelu and N. Akhmediev, Dissipative solitons for mode-locked lasers, Nat. Photon. 6, 84-92 (2012).

[63] P. Grelu, J. M. Soto-Crespo, N. Akhmediev, Light bullets and dynamic pattern formation in nonlinear dissipative systems, Opt. Express 13, 9352-9360 (2005).

[64] V. Skarka and N. B. Aleksić, Stability criterion for dissipative soliton solutions of the one-, two-, and three-dimensional complex cubic-quintic Ginzburg-Landau equations, Phys. Rev. Lett. 96, 013903 (2006).

[65] D. Mihalache, D. Mazilu, F. Lederer, Y. V. Kartashov, L. C. Crasovan, L. Torner, B. A. Malomed, Stable vortex tori in the three-dimensional cubic-quintic Ginzburg-Landau equation, Phys. Rev. Lett. 97, 073904 (2006).

[66] N. A. Veretenov, N. N. Rosanov, and S. V. Fedorov, Rotating and precessing dissipative-optical-topological-3D solitons, Phys. Rev. Lett. 117, 183901 (2016).

[67] N. A. Veretenov, S. V. Fedorov, and N. N. Rosanov, Topological vortex and knotted dissipative optical 3D solitons generated by 2D vortex solitons, Phys. Rev. Lett. 119, 263901 (2017).

[68] F. Gustave, N. Radwell, C. McIntyre, J. P. Toomey, D. M. Kane, S. Barland, W. J. Firth, G.-L. Oppo, and T. Ackemann, Observation of mode-locked spatial laser solitons, Phys. Rev. Lett. 118, 044102 (2017).

[69] O. Bang, W. Krolikowski, J. Wyller, and J. J. Rasmussen, Collapse arrest and soliton stabilization in nonlocal nonlinear media, Phys. Rev. E 66, 046619 (2002).

[70] C. Conti, M. Peccianti, and G. Assanto, Observation of optical spatial solitons in highly nonlocal medium, Phys. Rev. Lett. 92, 113902 (2004).

[71] C. Rotschild, O. Cohen, O. Manela, M. Segev, and T. Carmon, Solitons in nonlinear media with an infinite range of nonlocality: first observation of coherent elliptic solitons and of vortex-ring solitons, Phys. Rev. Lett. 95, 213904 (2005).

[72] I. B. Burgess, M. Peccianti, G. Assanto, and R. Morandotti, Accessible light bullets via synergetic nonlinearities, Phys. Rev. Lett. 102, 203903 (2009).

[73] O. Lahav, O. Kfir, P. Sidorenko, M. Mutzafi, A. Fleischer, and O. Cohen, Threedimensional spatiotemporal pulse-train solitons, Phys. Rev. X 7, 041051 (2017).

[74] J. Yang, Z. H. Musslimani, Fundamental and vortex solitons in a two-dimensional optical lattice, Opt. Lett. 28, 2094-2096 (2003).

[75] B. B. Baizakov, B. A. Malomed, and M. Salerno, Multidimensional solitons in periodic potentials. Europhys. Lett. 63, 642-648 (2003).

[76] N. K. Efremidis, J. Hudock, D. N. Christodoulides, J. W. Fleischer, O. Cohen, and M. Segev, Two-dimensional optical lattice solitons, Phys. Rev. Lett. 91, 213906 (2003).

[77] D. Mihalache, D. Mazilu, F. Lederer, Y.V. Kartashov, L.-C. Crasovan, L. Torner, Stable three-dimensional spatiotemporal solitons in a two-dimensional photonic lattice, Phys. Rev. E 70, 055603(R) (2004).

[78] B. B. Baizakov, B. A. Malomed and M. Salerno, Multidimensional solitons in a lowdimensional periodic potential, Phys. Rev. A 70, 053613 (2004).

[79] H. Leblond, B. A. Malomed, and D. Mihalache, Three-dimensional vortex solitons in quasi-two-dimensional lattices, Phys. Rev. E 76, 026604 (2007).

[80] D. N. Christodoulides, F. Lederer, Y. Silberberg, Discretizing light behavior in linear and nonlinear waveguide lattices, Nature 424, 817-823 (2003).

[81] J. W. Fleischer, M. Segev, N. K. Efremidis, D. N. Christodoulides, Observation of two-dimensional discrete solitons in optically induced nonlinear photonic lattices, Nature 422, 147-150 (2003).

[82] F. Lederer, G. I. Stegeman, D. N. Christodoulides, G. Assanto, M. Segev, and Y. Silberberg, Discrete solitons in optics, Phys. Rep. 463, 1-126 (2008).

[83] Y. V. Kartashov, V. A. Vysloukh, and L. Torner, Soliton shape and mobility control in optical lattices, Progr. Opt. 52, 63-148 (2009). 
[84] N. K. Efremidis, S. Sears, D. N. Christodoulides, J. W. Fleischer, and M. Segev, Discrete solitons in photorefractive optically induced photonic lattices, Phys. Rev. E 66, 046602 (2002).

[85] D. N. Neshev, T. J. Alexander, E. A. Ostrovskaya, Yuri S. Kivshar, H. Martin, I. Makasyuk, and Z. Chen, Observation of discrete vortex solitons in optically induced photonic lattices, Phys. Rev. Lett. 92, 123903 (2004).

[86] J. W. Fleischer, G. Bartal, O. Cohen, O. Manela, M. Segev, J. Hudock, and D. N. Christodoulides, Observation of vortex-ring discrete solitons in 2D photonic lattices, Phys. Rev. Lett. 92, 123904 (2004).

[87] B. Terhalle, T. Richter, A. S. Desyatnikov, D. N. Neshev, W. Krolikowski, F. Kaiser, C. Denz, and Y.S. Kivshar, Observation of multivortex solitons in photonic lattices, Phys. Rev. Lett. 101, 013903 (2008).

[88] Z. Chen, H. Martin, E. D. Eugenieva, J. Xu, and A. Bezryadina, Anisotropic enhancement of discrete diffraction and formation of two-dimensional discrete-soliton trains, Phys. Rev. Lett. 92, 143902 (2004).

[89] H. Martin, E. D. Eugenieva, Z. Chen, and D. N. Christodoulides, Discrete solitons and soliton-induced dislocations in partially coherent photonic lattices, Phys. Rev. Lett. 92, 123902 (2004).

[90] A. Szameit, J. Burghoff, T. Pertsch, S. Nolte, A. Tuennermann, F. Lederer, Twodimensional soliton in cubic fs laser written waveguide arrays in fused silica, Opt. Express 14, 6055-6062 (2006).

[91] A. Szameit and S. Nolte, Discrete optics in femtosecond-laser written photonic structures, J. Phys. B: At. Mol. Opt. Phys. 43, 163001 (2010).

[92] A. B. Aceves, C. De Angelis, A. M. Rubenchik, and S. K. Turitsyn, Multidimensional solitons in fiber arrays, Opt. Lett. 19, 329-331 (1994).

[93] A. B. Aceves, G. G. Luther, C. De Angelis, A. M. Rubenchik, and S. K. Turitsyn, Energy localization in nonlinear fibre arrays: Collapse-effect compressor, Phys. Rev. Lett. 75, $73-76$ (1995).

[94] D. E. Pelinovsky, Localization in Periodic Potentials (Cambridge University Press: Cambridge, 2011).

[95] S. Minardi, F. Eilenberger, Y. V. Kartashov, A. Szameit, U. Röpke, J. Kobelke, K. Schuster, H. Bartelt, S. Nolte, L. Torner, F. Lederer, A. Tünnermann, T. Pertsch, Threedimensional light bullets in arrays of waveguides, Phys. Rev. Lett. 105, 263901 (2010).

[96] F. Eilenberger, S. Minardi, A. Szameit, U. Röpke, J. Kobelke, K. Schuster, H. Bartelt, S. Nolte, L. Torner, F. Lederer, A. Tünnermann, and T. Pertsch, Evolution dynamics of discrete-continuous light bullets, Phys. Rev. A 84, 013836 (2011).

[97] F. Eilenberger, K. Prater, S. Minardi, R. Geiss, U. Röpke, J. Kobelke, K. Schuster, H. Bartelt, S. Nolte, A. Tünnermann, and T. Pertsch, Observation of discrete, vortex light bullets, Phys. Rev. X 3, 041031 (2013).

[98] D. Mihalache, D. Mazilu, F. Lederer, B. A. Malomed, Y. V. Kartashov, L. C. Crasovan, and L. Torner, Stable spatiotemporal solitons in Bessel optical lattices, Phys. Rev. Lett. 95, 023902 (2005).

[99] C. E. Rüter, K. G. Makris, R. El-Ganainy, D. N. Christodoulides, M. Segev, and D. Kip, Observation of parity-time symmetry in optics, Nat. Phys. 6, 192-195 (2010).

[100] Y. V. Kartashov, C. Hang, G. X. Huang, and L. Torner, Three-dimensional topological solitons in PT-symmetric optical lattices, Optica 3, 1048-1055 (2016).

[101] C. Conti, S. Trillo, P. Di Trapani, G. Valiulis, A. Piskarskas, O. Jedrkiewicz, and J. Trull, Nonlinear electromagnetic X waves, Phys. Rev. Lett. 90, 170406 (2003).

[102] P. Di Trapani, G. Valiulis, A. Piskarskas, O. Jedrkiewicz, J. Trull, C. Conti, and S. Trillo, Spontaneously generated X-shaped light bullets, Phys. Rev. Lett. 91, 093904 (2003).

[103] Y. Lahini, E. Frumker, Y. Silberberg, S. Droulias, K. Hizanidis, R. Morandotti, and D. N. Christodoulides, Discrete X-wave formation in nonlinear waveguide arrays, Phys. Rev. Lett. 98, 023901 (2007).

[104] M. Heinrich, A. Szameit, F. Dreisow, R. Keil, S. Minardi, T. Pertsch, S. Nolte, A. Tunnermann, and F. Lederer, Observation of three-dimensional discrete-continuous $X$ waves in photonic lattices, Phys. Rev. Lett. 103, 113903 (2009). 
[105] A. Chong, W. H. Renninger, D. N. Christodoulides, and F. W. Wise, Airy-Bessel wave packets as versatile linear light bullets, Nat. Photon. 4, 103-106 (2010).

[106] D. Abdollahpour, S. Suntsov, D. G. Papazoglou, and S. Tzortzakis, Spatiotemporal Airy light bullets in the linear and nonlinear regimes, Phys. Rev. Lett. 105, 253901 (2010).

[107] G. P. Agrawal, Nonlinear Fiber Optics (Academic Press, San Diego, 1995).

[108] S.-S. Yu, Ch.-H. Chien, Y. Lai, and J. Wang, Spatio-temporal solitary pulses in graded-index materials with Kerr nonlinearity. Opt. Commun. 119, 167-170 (1995).

[109] O. V. Shtyrina, M. P. Fedoruk, Y. S. Kivshar, and S. K. Turitsyn, Coexistence of collapse and stable spatiotemporal solitons in multimode fibers, Phys. Rev. A 97, 013841 (2018).

[110] W. H. Renninger and F. W. Wise, Optical solitons in graded-index multimode fibres, Nat. Commun. 4, 1719 (2013).

[111] L. G. Wright, D. N. Christodoulides, and F. Wise, Controllable spatiotemporal nonlinear effects in multimode fibres, Nature Photonics 9, 306-310 (2015).

[112] L. G. Wright, S. Wabnitz, D. N. Christodoulides, F. W. Wise, Ultrabroadband dispersive radiation by spatiotemporal oscillation of multimode waves, Phys. Rev. Lett. 115, 223902 (2015).

[113] L. G. Wright, Z. Liu, D. A. Nolan, M.-J. Li, D. N. Christodoulides, and F. W. Wise, Self-organized instability in graded-index multimode fibres, Nature Photonics 10, 771-776 (2016).

[114] K. Krupa, A. Tonello, B. M. Shalaby, M. Fabert, A. Barthélémy, G. Millot, S. Wabnitz, and V. Couderc, Spatial beam self-cleaning in multimode fibres, Nature Photonics 11, 237-242 (2017).

[115] J. Kasprzak, M. Richard, S. Kundermann, A. Baas, P. Jeambrun, J. M. J. Keeling, F. M. Marchetti, M. H. Szymanska, R. Andre, J. L. Staehli, V. Savona, P. B. Littlewood, B. Deveaud, and Le Si Dang, Bose-Einstein condensation of exciton polaritons, Nature 443, 409-414 (2006).

[116] A. Amo, S. Pigeon, D. Sanvitto, V. G. Sala, R. Hivet, I. Carusotto, F. Pisanello, G. Lemenager, R. Houdre, E. Giacobino, C. Ciuti, A. Bramati, Polariton superfluids reveal quantum hydrodynamic solitons, Science 332, 1167-1170 (2011).

[117] G. Nardin, G. Grosso, Y. Leger, B. Pietka, F. Morier-Genoud, D. DeveaudPledran, Hydrodynamic nucleation of quantized vortex pairs in a polariton quantum fluid, Nat. Phys. 7, 635-641 (2011).

[118] O. A. Egorov, D. V. Skryabin, A. V. Yulin, and F. Lederer, Bright cavity polariton solitons, Phys. Rev. Lett. 102, 153904 (2009).

[119] M. Sich, D. N. Krizhanovskii, M. S. Skolnick, A. V. Gorbach, R. Hartley, D. V. Skryabin, E. A. Cerda-Mendez, K. Biermann, R. Hey, and P. V. Santos, Observation of bright polariton solitons in a semiconductor microcavity, Nat. Photon. 6, 50-55 (2012).

[120] V. G. Sala, D. D. Solnyshkov, I. Carusotto, T. Jacqmin, A. Lemaitre, H. Terças, A. Nalitov, M. Abbarchi, E. Galopin, I. Sagnes, J. Bloch, G. Malpuech, and A. Amo, Spin-orbit coupling for photons and polaritons in microstructures, Phys. Rev. X 5, 011034 (2015).

[121] C. Schneider, K. Winkler, M. D. Fraser, M. Kamp, Y. Yamamoto, E. A. Ostrovskaya, S. Hofling, Exciton-polariton trapping and potential landscape engineering, Rep. Prog. Phys. 80, 016503 (2017).

[122] D. Tanese, H. Flayac, D. Solnyshkov, A. Amo, A. Lemaitre, E. Galopin, R. Braive, P. Senellart, I. Sagnes, G. Malpuech, J. Bloch, Polariton condensation in solitonic gap states in a one-dimensional periodic potential, Nat. Commun. 4, 1749 (2013).

[123] E. A. Cerda-Mendez, D. Sarkar, D. N. Krizhanovskii, S. S. Gavrilov, K. Biermann, M. S. Skolnick, P. V. Santos, Exciton-polariton gap solitons in two-dimensional lattices, Phys. Rev. Lett. 111, 146401 (2013).

[124] A. V. Gorbach, B. A. Malomed, and D. V. Skryabin, Gap polariton solitons, Phys. Lett. A 373, 3024-3027 (2009).

[125] E. A. Ostrovskaya, J. Abdullaev, M. D. Fraser, A. S. Desyatnikov, and Y. S. Kivshar, Self-localization of polariton condensates in periodic potentials, Phys. Rev. Lett. 110, 170407 (2013). 
[126] A. V. Nalitov, D. D. Solnyshkov, and G. Malpuech, Polariton Z topological insulator, Phys. Rev. Lett. 114, 116401 (2015).

[127] Y. V. Kartashov and D. V. Skryabin, Modulational instability and solitary waves in polariton topological insulators, Optica 3, 1228-1236 (2016).

[128] S. Tsesses, E. Ostrovsky, K. Cohen, B. Gjonaj, N. H. Lindner, and G. Bartal, Optical skyrmion lattice in evanescent electromagnetic fields, Science 361, 993-996 (2018).

[129] A. Couairon, A Mysyrowicz, Femtosecond filamentation in transparent media, Phys. Rep. 441, 47-189 (2007).

[130] M. Durand, A. Jarnac, A. Houard, Y. Liu, S. Grabielle, N. Forget, A. Durecu, A. Couairon, and A. Mysyrowicz, Self-guided propagation of ultrashort laser pulses in the anomalous dispersion region of transparent solids: a new regime of filamentation, Phys. Rev. Lett. 110, 115003 (2013).

[131] S. V. Chekalin, A. E. Dokukina, A. E. Dormidonov, V. O. Kompanets, E. O. Smetanina, and V. P. Kandidov, Light bullets from a femtosecond filament, J. Phys. B: At. Mol. Opt. Phys. 48, 094008 (2015).

[132] S. V. Chekalin, V. O. Kompanets, A. E. Dormidonov, V. P. Kandidov, Path length and spectrum of single-cycle mid-IR light bullets in transparent dielectrics, Quantum Electronics 48, 372-377 (2018).

[133] D. Majus, D. Majus, G. Tamošauskas, I. Gražulevičiūtè, N. Garejev, A. Lotti, A. Couairon, D. Faccio, and A. Dubietis, Nature of spatiotemporal light bullets in bulk Kerr media, Phys. Rev. Lett. 112, 193901 (2014).

[134] M. Scheller, M. S. Mills, M.-A. Miri, W. Cheng, J. V. Moloney, M. Kolesik, P. Polynkin, D. N. Christodoulides, Externally refueled optical filaments, Nature Photonics 8, 297 (2014).

[135] P. Panagiotopoulos, P. Whalen, M. Kolesik, and J. V. Moloney, Super high power mid-infrared femtosecond light bullet, Nat. Photon. 9, 543-548 (2015).

[136] O. V. Borovkova, Y. V. Kartashov, B. A. Malomed, and L. Torner, Algebraic bright and vortex solitons in defocusing media, Opt. Lett. 36, 3088-3090 (2011).

[137] O. V. Borovkova, Y. V. Kartashov, L. Torner, and B. A. Malomed, Bright solitons from defocusing nonlinearities, Phys. Rev. E 84, 035602(R) (2011).

[138] Q. Tian, L. Wu, Y. Zhang, and J.-F. Zhang, Vortex solitons in defocusing media with spatially inhomogeneous nonlinearity, Phys. Rev. E 85, 056603 (2012).

[139] Y. Wu, Q. Xie, H. Zhong, L. Wen, and W. Hai, Algebraic bright and vortex solitons in self-defocusing media with spatially inhomogeneous nonlinearity, Phys. Rev. A 87, 055801 (2013).

[140] R. Driben, Y. V. Kartashov, B. A. Malomed, T. Meier, and L. Torner, Soliton gyroscopes in media with spatially growing repulsive nonlinearity, Phys. Rev. Lett. 112, 020404 (2014).

[141] Y. V. Kartashov, B. A. Malomed, Y. Shnir, and L. Torner, Twisted toroidal vortexsolitons in inhomogeneous media with repulsive nonlinearity, Phys. Rev. Lett. 113, 264101 (2014).

[142] R. Driben, Y. Kartashov, B. A. Malomed, T. Meier, and L. Torner, Threedimensional hybrid vortex solitons, New J. Phys. 16, 063035 (2014).

[143] Y. V. Kartashov, B. A. Malomed, and L. Torner, Solitons in nonlinear lattices, Rev. Mod. Phys. 83, 247-306 (2011).

[144] G. Roati, M. Zaccanti, C. D'Errico, J. Catani, M. Modugno, A. Simoni, M. Inguscio, and G. Modugno, ${ }^{39} \mathrm{~K}$ Bose-Einstein condensate with tunable interactions, Phys. Rev. Lett. 99, 010403 (2007).

[145] S. E. Pollack, D. Dries, M. Junker, Y. P. Chen, T. A. Corcovilos, and R. G. Hulet, Extreme tunability of interactions in a ${ }^{7} \mathrm{Li}$ Bose-Einstein condensate, Phys. Rev. Lett. 102, 090402 (2009).

[146] P. O. Fedichev, Yu. Kagan, G. V. Shlyapnikov, and J. T. M. Walraven, Influence of nearly resonant light on the scattering length in low-temperature atomic gases, Phys. Rev. Lett. 77, 2913-2916 (1996).

[147] M. Yan, B. J. DeSalvo, B. Ramachandhran, H. Pu, and T. C. Killian, Controlling condensate collapse and expansion with an optical Feshbach resonance, Phys. Rev. Lett. 110, 123201 (2013). 
[148] R. Yamazaki, S. Taie, S. Sugawa, and Y. Takahashi, Submicron spatial modulation of an interatomic interaction in a Bose-Einstein condensate, Phys. Rev. Lett. 105, 050405 (2010).

[149] L. W. Clark, L.-C. Ha, C.-Y. Xu, and C. Chin, Quantum dynamics with spatiotemporal control of interactions in a stable Bose-Einstein condensate, Phys. Rev. Lett. 115, 155301 (2015).

[150] J. Hukriede, D. Runde, and D. Kip, Fabrication and application of holographic Bragg gratings in lithium niobate channel waveguides, J. Phys. D 36, R1-R156 (2003).

[151] O. Morsch and M. Oberthaler, Dynamics of Bose-Einstein condensates in optical lattices, Rev. Mod. Phys. 78, 179-215 (2006).

[152] I. Bloch, J. Dalibard, and W. Zwerger, Many-body physics with ultracold gases, Rev. Mod. Phys. 80, 885-964 (2008).

[153] N. Goldman, J. C. Budich, and P. Zoller, Topological quantum matter with ultracold gases in optical lattices, Nature Phys. 12, 639-645 (2016).

[154] E. A. Ostrovskaya and Y. S. Kivshar, Matter-wave gap solitons in atomic band-gap structures, Phys. Rev. Lett. 90, 160407 (2003).

[155] P. J. Y. Louis, E. A. Ostrovskaya, C. M. Savage, and Y. S. Kivshar, Bose-Einstein condensates in optical lattices: Band-gap structure and solitons, Phys. Rev. A 67, 013602 (2003).

[156] E. A. Ostrovskaya and Y. S. Kivshar, Matter-wave gap vortices in optical lattices, Phys. Rev. Lett. 93, 160405 (2004).

[157] H. Sakaguchi and B. A. Malomed, Two-dimensional loosely and tightly bound solitons in optical lattices and inverted traps, J. Phys. B 37, 2225-2239 (2004).

[158] Y. V. Kartashov, V. A. Vysloukh, and L. Torner, Rotary solitons in Bessel optical lattices, Phys. Rev. Lett. 93, 093904 (2004).

[159] B. Baizakov, B. A. Malomed, and M. Salerno, Matter-wave solitons in radially periodic potentials, Phys. Rev. E 74, 066615 (2006).

[160] Y.-J. Lin, K. Jiménez-García, I. B. Spielman, Spin-orbit-coupled Bose-Einstein condensates, Nature 471, 83-86 (2011).

[161] H. Zhai, Degenerate quantum gases with spin-orbit coupling: A review, Rep. Prog. Phys. 78, 026001 (2015).

[162] H. Sakaguchi, B. Li, B. A. Malomed, Creation of two-dimensional composite solitons in spin-orbit-coupled self-attractive Bose-Einstein condensates in free space, Phys. Rev. E 89, 032920 (2014).

[163] L. Salasnich, W. B. Cardoso, and B. A. Malomed, Localized modes in quasi-twodimensional Bose-Einstein condensates with spin-orbit and Rabi couplings, Phys. Rev. A 90, 033629 (2014).

[164] H. Sakaguchi, E. Ya. Sherman, and B. A. Malomed, Vortex solitons in twodimensional spin-orbit coupled Bose-Einstein condensates: Effects of the Rashba-Dresselhaus coupling and the Zeeman splitting, Phys. Rev. E 94, 032202 (2016).

[165] Y.-C. Zhang, Z.-W. Zhou, B. A. Malomed, H. Pu, Stable solitons in three dimensional free space without the ground state: Self-trapped Bose-Einstein condensates with spinorbit coupling, Phys. Rev. Lett. 115, 253902 (2015).

[166] J. Qin, G. Dong, and B. A. Malomed, Stable giant vortex annuli in microwavecoupled atomic condensates, Phys. Rev. A 94, 053611 (2016).

[167] A. Bulgac, Dilute Quantum Droplets, Phys. Rev. Lett. 89, 050402 (2002).

[168] G. E. Volovik, The universe in a helium droplet, Oxford University Press, Oxford (2009).

[169] D. S. Petrov, Liquid beyond the van der Waals paradigm, Nature Physics 14, 211 (2018).

[170] A. Cappellaro, T. Macrí, G. F. Bertacco, and L. Salasnich, Equation of state and self-bound droplet in Rabi-coupled Bose mixtures, Sci. Rep. 7, 13358 (2017).

[171] X. Cui, Spin-orbit coupling induced quantum droplet in ultracold Bose-Fermi mixtures, Phys. Rev. A 98, 023630 (2018).

[172] H. Saito, Path-Integral Monte Carlo Study on a Droplet of a Dipolar Bose-Einstein Condensate Stabilized by Quantum Fluctuation, J. Phys. Soc. Jpn. 85, 053001 (2016).

[173] V. Cikojević, K. Dželalija, P. Stipanović, L. Vranješ Markić, and J. Boronat, Ultradilute quantum liquid drops, Phys. Rev. B 97, 140502(R) (2018). 
[174] V. Cikojević, L. Vranješ Markić, G. E. Astrakharchik and J. Boronat, Universality in ultradilute liquid Bose-Bose mixtures, arXiv:1811.04436.

[175] C. Staudinger, F. Mazzanti, R. E. Zillich, Self-bound Bose mixtures, Phys. Rev. A 98, 023633 (2018).

[176] Y. Li, Z. Chen, Z. Luo, C. Huang, H. Tan, W.i Pang, and B. A. Malomed, Twodimensional vortex quantum droplets, Phys. Rev. A 98, 063602 (2018).

[177] G. E. Astrakharchik, B. A. Malomed, Dynamics of one-dimensional quantum droplets, Phys. Rev. A 98, 013612 (2018).

[178] F. Wächtler and L. Santos, Ground-state properties and elementary excitations of quantum droplets in dipolar Bose-Einstein condensates, Phys. Rev. A 94, 043618 (2016).

[179] K.-T. Xi and H. Saito, Droplet formation in a Bose-Einstein condensate with strong dipole-dipole interaction, Phys. Rev. A 93, 011604(R) (2016).

[180] S. K. Adhikari, Statics and dynamics of a self-bound dipolar matter-wave droplet, Laser Phys. Lett. 14, 025501 (2017).

[181] D. Edler, C. Mishra, F. Wächtler, R. Nath, S. Sinha, and L. Santos, Quantum fluctuations in quasi-one-dimensional dipolar Bose-Einstein condensates, Phys. Rev. Lett. 119, 050403 (2017).

[182] F. Wächtler and L. Santos, Quantum filaments in dipolar Bose-Einstein condensates, Phys. Rev. A 93, 061603(R) (2016).

[183] T. Koch, T. Lahaye, J. Metz, B. Fröhlich, A. Griesmaier, and T. Pfau, Stabilization of a purely dipolar quantum gas against collapse, Nat. Phys. 4, 218-222 (2008).

[184] A. Cidrim, F. E. A. dos Santos, E. A. L. Henn, and T. Macrí, Vortices in self-bound dipolar droplets, Phys. Rev. A 98, 023618 (2018).

[185] Y. V. Kartashov, B. A. Malomed, L. Tarruell, and L. Torner, Three-dimensional droplets of swirling superfluids, Phys. Rev. A 98, 013612 (2018).

[186] P. J. Ackerman and I. I. Smalyukh, Diversity of knot solitons in liquid crystals manifested by linking of preimages in torons and hopfions, Phys. Rev. X 7, 011006 (2017).

[187] P. J. Ackerman and I. I. Smalyukh, Static three-dimensional topological solitons in fluid chiral ferromagnets and colloids, Nature Materials 16, 426-432 (2017).

[188] J.-S. Tai, P. I. Ackerman, and I. I. Smalyukh, Topological transformations of Hopf solitons in chiral ferromagnets and liquid crystals, Proc. Nat. Acad. Sci. 115, 921-926 (2018).

[189] R. H. Hobart, On the instability of a class of unitary field models, Proc. Phys. Soc. London 82, 201-203 (1963).

[190] G. H. Derrick, Comments on nonlinear wave equations as models for elementary particles, J. Math. Phys. 5, 1252-1254 (1964).

[191] B.-X. Li, V. Borshch, R.-L. Xiao, S. Paladugu, T. Turiv, S. V. Shiyanovskii, and O. D. Lavrentovich, Electrically driven three-dimensional solitary waves as director bullets in nematic liquid crystals, Nature Comm. 9, 2912 (2018).

[192] P. Lai, G. P. Zhao, H. Tang, N. Ran, S. Q. Wu, J. Xia, X. Zhang, and Y. Zhou, An improved racetrack structure for transporting a skyrmion, Sci. Rep. 7, 45330 (2017).

[193] P. J. Ackerman, T. Boyle, and I. I. Smalyukh, Squirming motion of baby skyrmions in nematic fluids, Nature Commun. 8, 673 (2017).

[194] D.-L. Deng, S.-T. Wang, K. Sun, and L.-M. Duan, Probe knots and Hopf insulators with ultracold atoms, Chin. Phys. Lett. 35, 013701 (2018).

[195] T. J. Kippenberg, A. L. Gaeta, M. Lipson, and M. L. Gorodetsky, Dissipative Kerr solitons in optical microcavities, Science 361, 567 (2018). 


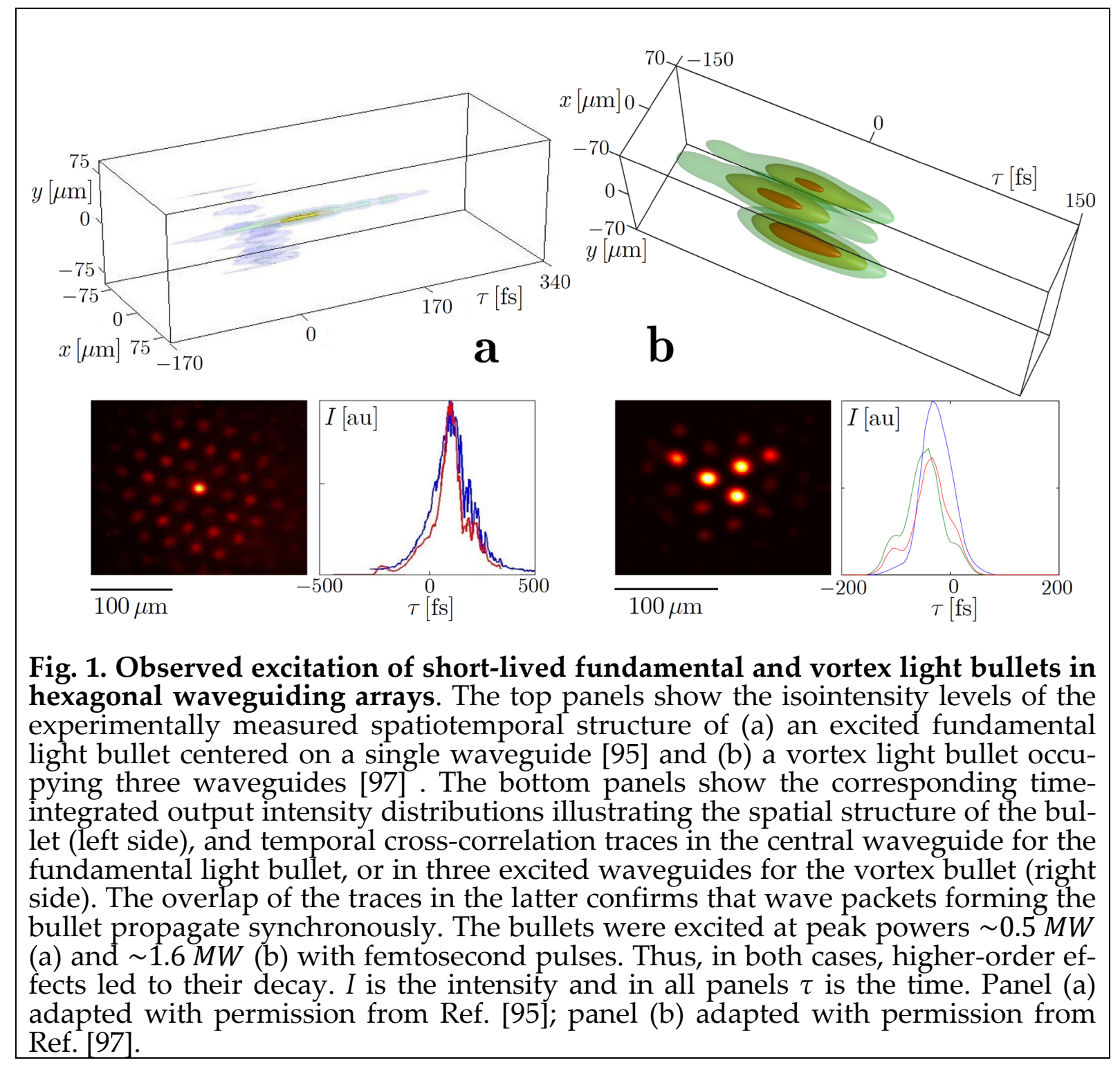




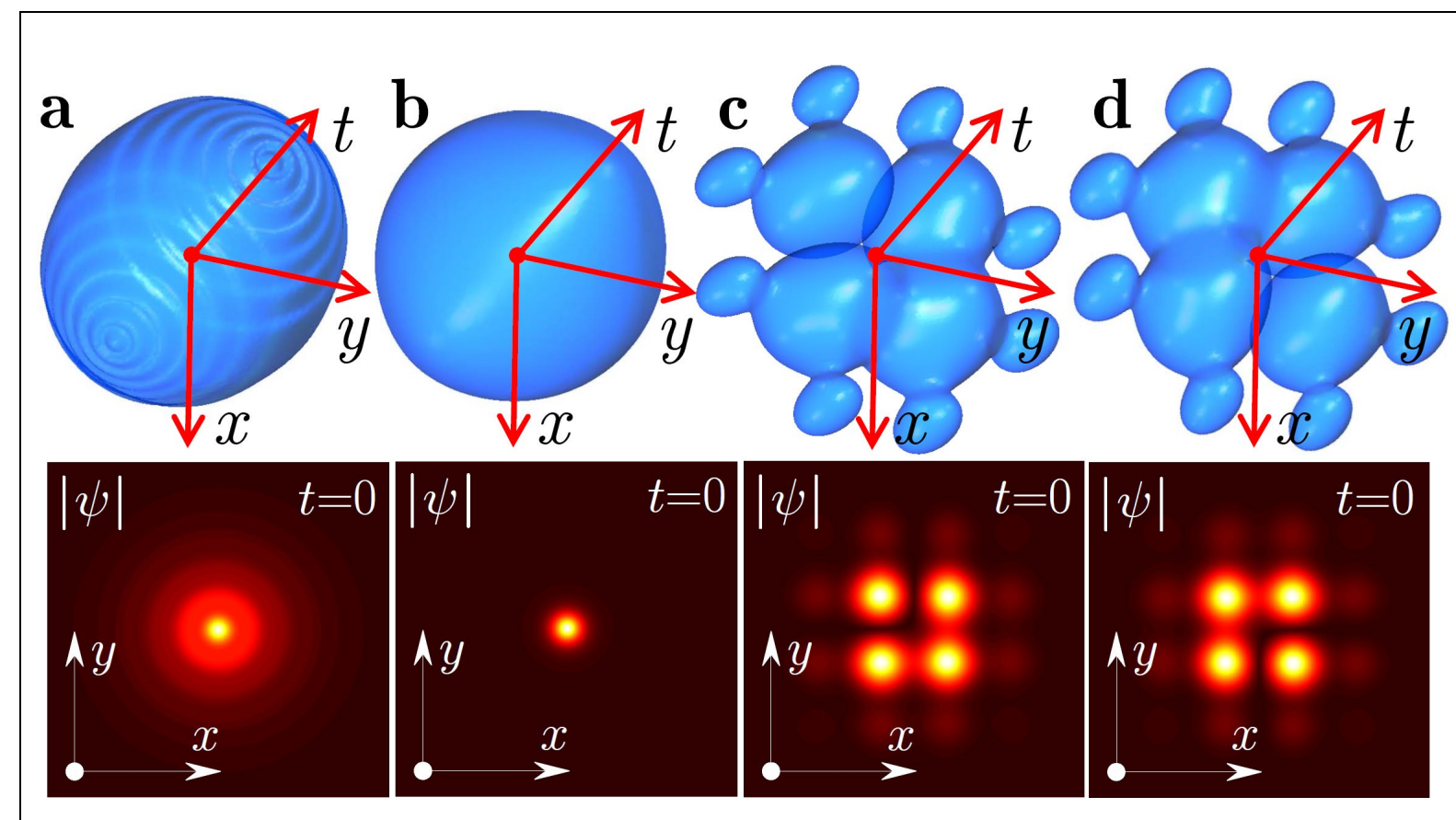

Fig. 2. Predicted light bullets in radially-symmetric and complex potentials. Isosurface plots (top) and spatial distributions of the field's absolute value at $t=0$ (bottom) show extended (panel a) and strongly localized (panel b) stable fundamental bullets supported by the two-dimensional Bessel lattice, as in Ref. [98], as well as vortex bullets with topological charges +1 (panel c) and -1 (panel d), supported by a two-dimensional periodic $\mathcal{P} \mathcal{T}$-symmetric lattice, as in Ref. [100]. Note the symmetry imposed by the lattice potential on the spatial profile of the bullets. In the $\mathcal{P} \mathcal{T}$-symmetric lattice, the shape of the bullet depends on the sign of its topological charge. Panels (a),(b) adapted with permission from Ref. [98]; panels (c),(d) reproduced with permission from Ref. [100]. 


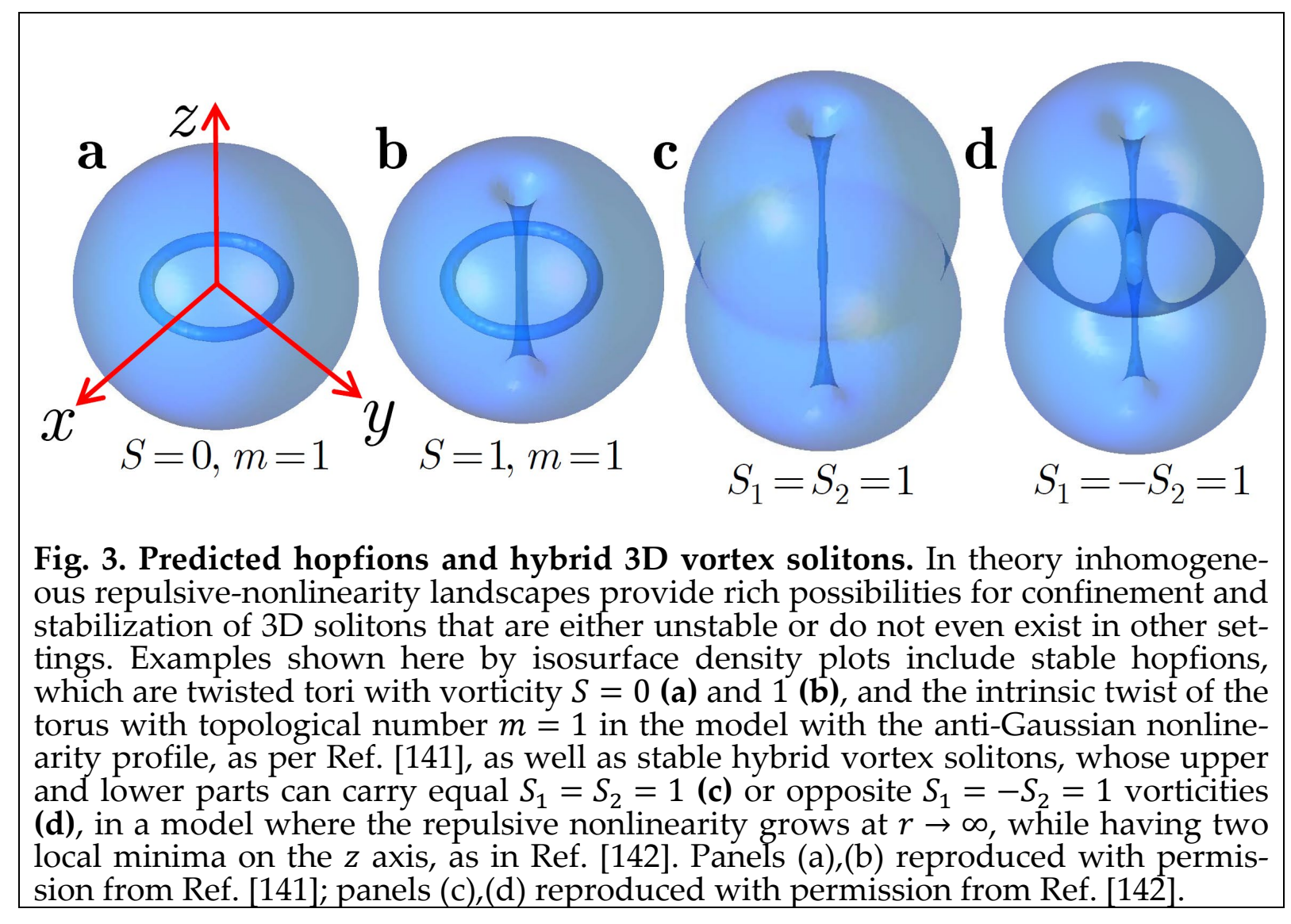




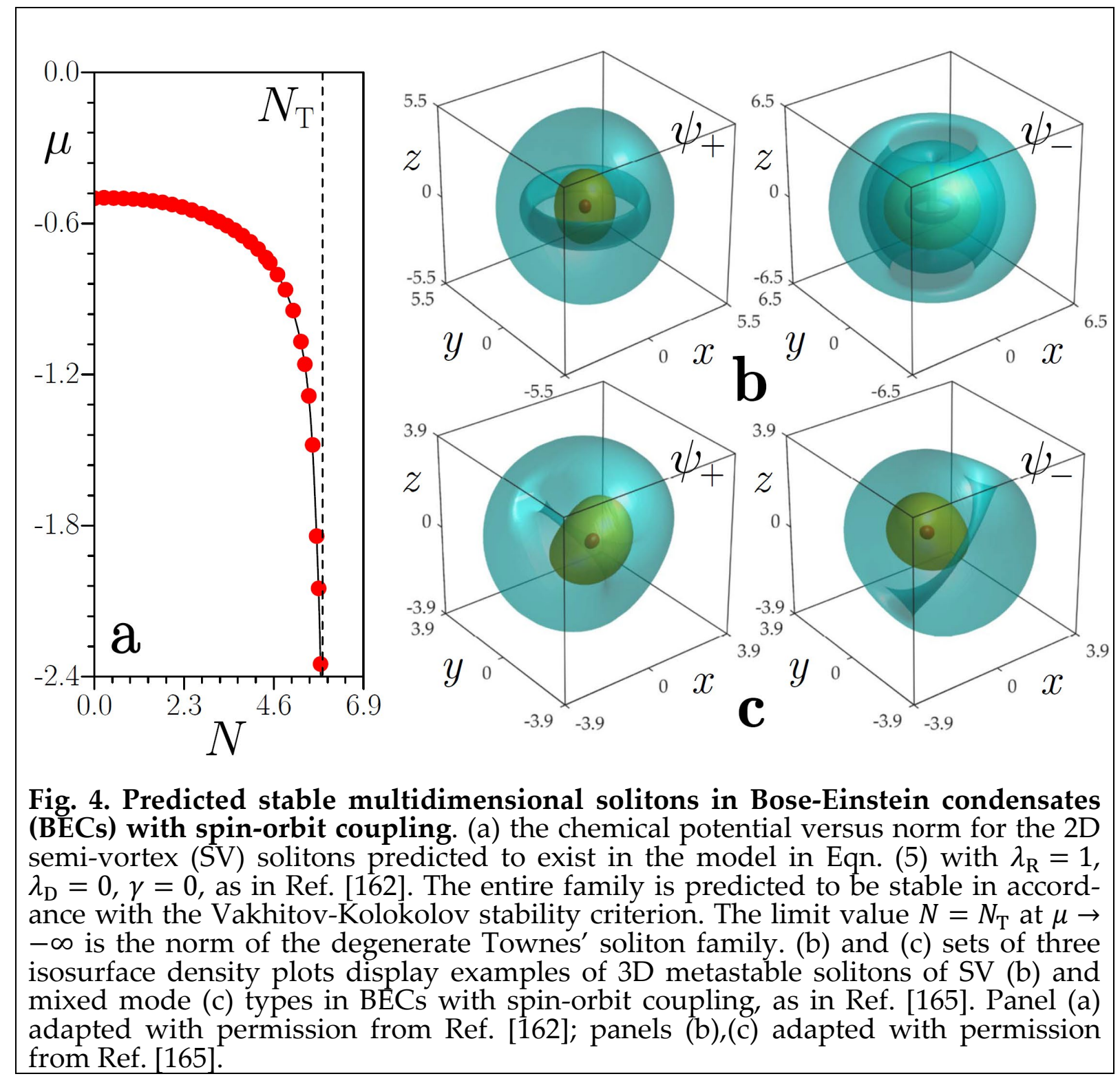




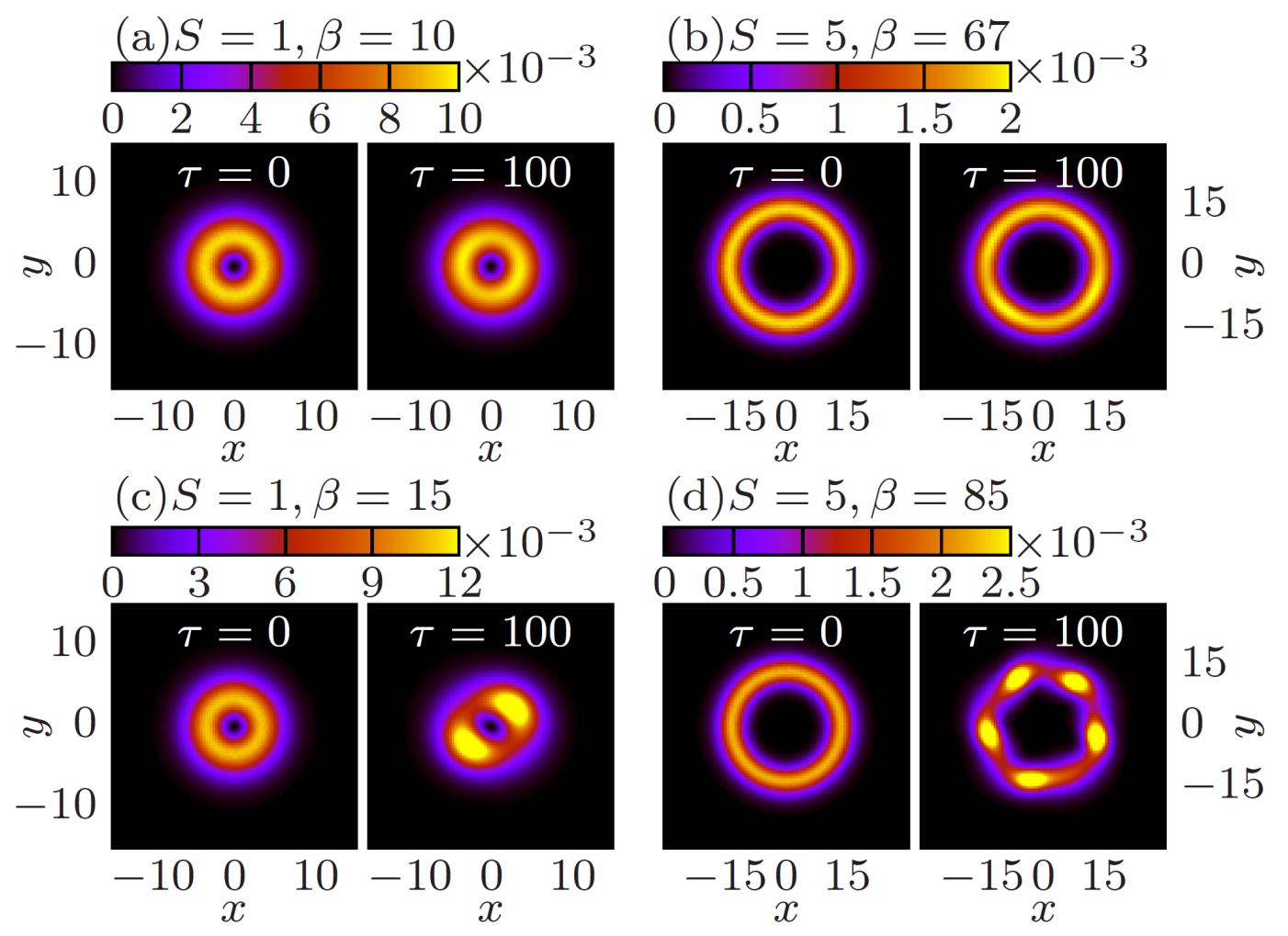

Fig. 5. Predicted vortex solitons in the binary Bose-Einstein condensates coupled by a microwave field. Stable (panel a and b) and unstable (panel $c$ and $d$ ) evolution of the hybrid vortex solitons with topological charges $S=1$ and 5 ( $\beta$ is the strength of the attractive contact interaction, see Eqn. (5)). The unstable solutions are predicted to split into necklace-like patterns, which may keep their shape for a while in the course of the subsequent evolution [166]. Figure reproduced with permission from Ref. [166]. 


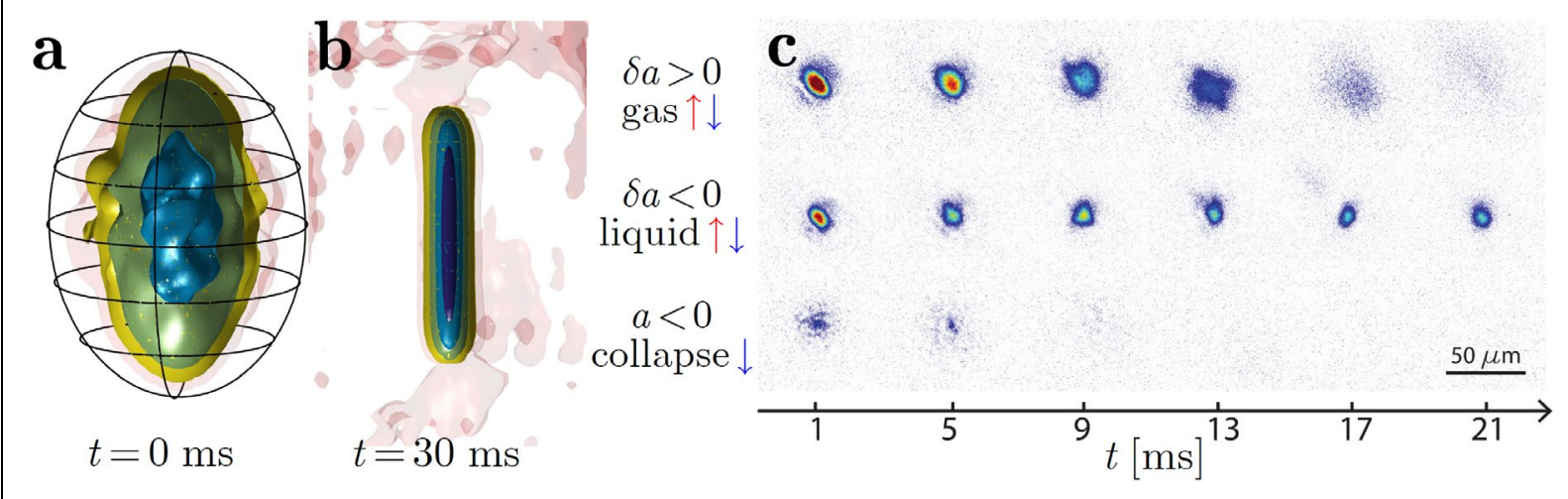

Fig. 6. Observed self-sustained multidimensional quantum droplets. Dipolar droplets that were predicted to form in the condensate of ${ }^{164} \mathrm{Dy}$ atoms [23] before (a) and after (b) turning off the trapping potential and adjusting the s-wave scattering length. The black lines show the shape of the external trap when it is present. (c) Experimental observation of quantum droplets in a two-component condensate of ${ }^{39} \mathrm{~K}$ atoms [27]: expansion of a two-component gas (top row), stable self-bound evolution of two-component liquid (middle row) and collapse of a single attractive component (bottom row). The sign of the scattering length $\delta a=a_{\uparrow \downarrow}+\left(a_{\uparrow \uparrow} a_{\downarrow \downarrow}\right)^{1 / 2}$ is indicated for each row, where $a_{\uparrow \uparrow}, a_{\downarrow \downarrow}$ and $a_{\uparrow \downarrow}$ are intra- and inter-state scattering lengths (the state is indicated by arrows $\uparrow$ or $\downarrow$ ), $a$ is the scattering length for singlecomponent condensate. The interplay between the mean-field interactions and quantum fluctuations stabilizes the droplets. Panels (a),(b) reproduced with permission from Ref. [23]; panel (c) reproduced with permission from Ref. [27]. 


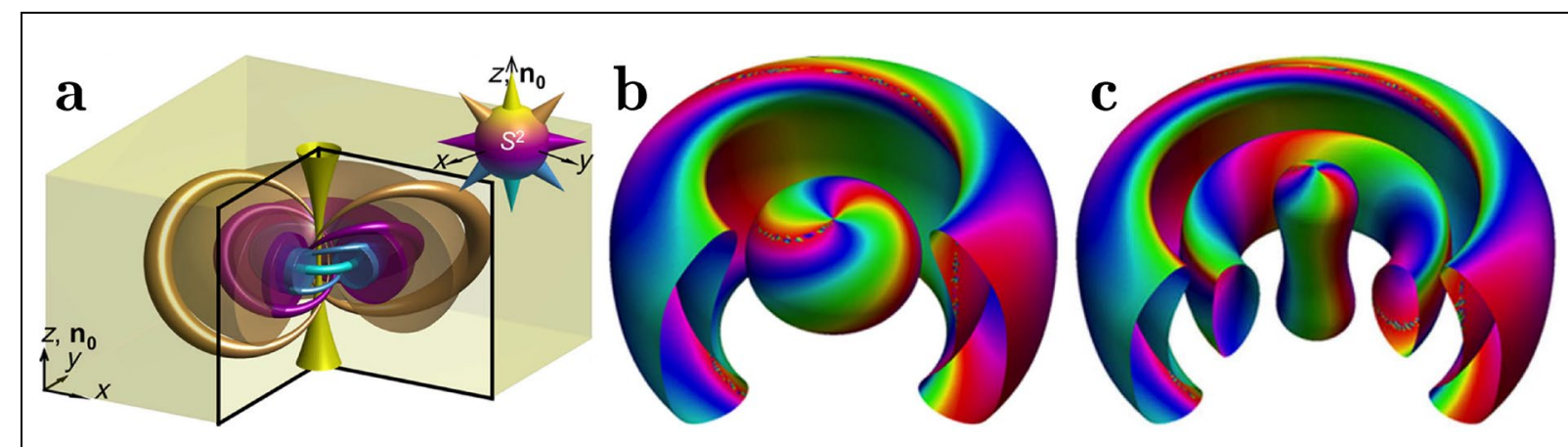

Fig. 7. Observed topological solitons with differently knotted nematic fields in a liquid crystal. (a) A closed-loop preimage (regions in the 3D liquid-crystal sample that have the same orientation of the physical field), and isosurfaces drawn at $n_{z}=$ 0 , illustrating the structure of $3 \mathrm{D}$ torons with complex linking, and $3 \pi$ (b) or $5 \pi$ (c) twist between their central axes and the far-field periphery, as in Ref. [186]. $S^{2}$ is (a) is the two-dimensional sphere used to define the core of the preimage. Surface colours denote different azimuthal orientations in the $(x, y)$ plane of the nematic director $\mathbf{n}(\mathbf{r})$ in the topological soliton. Figure reproduced with permission from Ref. [186]. 


\section{Glossary}

\section{Feshbach resonance}

The effect which makes it possible to change the magnitude and sign of the scattering length characterizing collisions between atoms in quantum gases. It is a powerful experimental tool enabling the control of the strength and sign of the effective nonlinearity in Bose-Einstein condensates (as it concerns both self- and cross- interactions, in the case of two-component condensates).

\section{hopfion}

A class of 3D localized modes, in the form of tori carrying the global vorticity, which are additionally twisted in the torus' cross section. This mode carries two independent topological charges (winding numbers), one representing the overall vorticity and the other accounting for the intrinsic twist.

\section{mixed modes}

Stable 2D and 3D solitons in two-component Bose-Einstein condensate with the spinorbit coupling between the components. Unlike semi-vortices, each component of such a mode is a mixture of terms with zero vorticity, and vorticities (+/-)1.

\section{PT symmetry}

The special symmetry of evolution equation or non-Hermitian Hamiltonian governing dissipative system under the transformation of time reversal and parity inversion (flip of the sign of spatial coordinate). In the so-called unbroken PT phase, such Hamiltonian shows an entirely real energy spectrum in spite of being non-Hermitian.

\section{pseudopotential}

An effective potential which is induced by the nonlinearity whose local strength is subject to spatial modulation.

\section{skyrmions}

Complex 3D states in various two-component field-theory systems, which carry two independent topological numbers. They were introduced by Skyrme as a classical-field model which effectively describes barions, and may be derived as a low-energy (semi-classical) limit of quantum chromodynamics.

\section{semi-vortex}

A stable two-component 2D or 3D soliton in two-component spin-orbit-coupled Bose-Einstein condensate, in which, unlike mixed modes, one component has zero vorticity, whereas the other one carries vorticity 1.

spinor Bose-Einstein condensate

A condensate composed of two or several components, which may be considered as a set forming a spinor wave function, corresponding to pseudo spin 1/2 (two components), 1 (three components), or 2 (five components).

\section{spin-orbit coupling}

Originally it referred to the coupling between the spin of electrons

in semiconductors and their motion through the crystalline electrostatic field. In the context of Bose-Einstein condensate, spin-orbit coupling is realized as linear mixing between two components

of a binary condensate through first spatial derivatives of the respective wave functions.

\section{topological insulator}

Originally, a dielectric material (insulator) possessing complete gap in the bulk, 
but admitting conductance through in-gap edge states, existing due to peculiarities of the intrinsic topological structure of the material. This

name is also used for photonic settings which emulate the same phenomenology in terms of light transmission.

\section{toron}

A toroidal localized mode which may be created in liquid crystals and ferrofluids. It is organized in essentially the same way as a hopfion (a twisted torus, which may carry overall vorticity).

\section{vortex soliton}

A 2D or 3D soliton represented by a complex wave function whose phase carries an integer winding number (vorticity, alias the topological charge), and has the amplitude vanishing at the central pivot.

\section{X-wave}

A delocalized linear or nonlinear 2D wave with the local power featuring an $X$ shape profile, which may be supported by defocusing nonlinear optical material when signs of dispersion and diffraction coefficients are opposite.

\section{Bessel and Airy beams}

Bessel beams represent two-dimensional nondiffracting solutions of the Helmholtz equation in circular cylindrical coordinates, where this equation is separable. Airy beams are nondiffracting one- or two-dimensional beams that bend along parabolic trajectory upon propagation, while maintaining their functional shapes. Their combinations can be used to construct nondiffracting three-dimensional wavepackets.

\section{Tandem structures}

Optical tandem structures represent periodic stacks of materials with different parameters, such as refractive index, nonlinearity, and dispersion, where widths of individual layers are usually small in comparison with average diffraction and dispersion lengths.

\section{Kerr nonlinearity}

Universal optical nonlinearity occurring in dielectric media that yields a correction to the local refractive index proportional to the intensity of the electromagnetic wave. It is represented by the cubic self-attractive term in the corresponding nonlinear Schrödinger equation. 